\title{
LEMUR: Large European Module for solar Ultraviolet
} Research

\section{European contribution to JAXA's Solar-C mission}

Luca Teriaca - Vincenzo Andretta - Frédéric Auchère - Charles M. Brown · Eric Buchlin . Gianna Cauzzi · J. Len Culhane · Werner Curdt · Joseph M. Davila · Giulio Del Zanna · George A. Doschek · Silvano Fineschi · Andrzej Fludra · Peter T. Gallagher · Lucie Green · Louise K. Harra · Shinsuke Imada . Davina Innes · Bernhard Kliem · Clarence Korendyke · John T. Mariska · Valentin Martínez-Pillet · Susanna Parenti · Spiros Patsourakos - Hardi Peter . Luca Poletto . Rob Rutten · Udo Schühle · Martin Siemer . Toshifumi Shimizu · Hector Socas-Navarro . Sami K. Solanki · Daniele Spadaro · Javier Trujillo-Bueno · Saku Tsuneta · Santiago Vargas Dominguez · Jean-Claude Vial · Robert Walsh · Harry P. Warren - Thomas Wiegelmann · Berend Winter · Peter Young

Received: date / Accepted: date

L. Teriaca, W. Curdt, D. Innes, H. Peter, U. Schühle, S. K. Solanki, T. Wiegelmann Max Planck Institute for Solar System Research

Max Planck str. 2, 37191 Katlenburg-Lindau, Germany

E-mail: teriaca@mps.mpg.de

V. Andretta

INAF - Osservatorio Astronomico di Capodimonte, Salita Moiariello 16, 80131 Napoli, Italy

F. Auchère, E. Buchlin, J.-C. Vial

Institut d'Astrophysique Spatiale, Bâtiment 121, Université Paris-Sud 11, 91405 Orsay, France

C.M. Brown, G.A. Doschek, C. Korendyke, J.T. Mariska, H.P. Warren

Space Science Division, Naval Research Laboratory, Washington DC, 20375-5320, USA

G. Cauzzi

INAF - Osservatorio Astrofisico di Arcetri, 50125 Florence, Italy

J.L. Culhane, L. Green, L.K. Harra, S. Vargas Dominguez, B. Winter

UCL - Mullard Space Science Laboratory, Holmbury St. Mary, Dorking, Surrey RH5 6NT, UK

J.M. Davila

NASA - Goddard Space Flight Center, Greenbelt, MD. USA

G. Del Zanna 
Abstract The solar outer atmosphere is an extremely dynamic environment characterized by the continuous interplay between the plasma and the magnetic field that generates and permeates it. Such interactions play a fundamental role in hugely diverse astrophysical systems, but occur at scales that cannot be studied outside the solar system.

Understanding this complex system requires concerted, simultaneous solar observations from the visible to the vacuum ultraviolet (VUV) and soft X-rays, at high spatial resolution (between $0.1^{\prime \prime}$ and $0.3^{\prime \prime}$ ), at high temporal resolution (on the order of $10 \mathrm{~s}$, i.e., the time scale of chromospheric dynamics), with a wide temperature coverage (0.01 MK to $20 \mathrm{MK}$, from the chromosphere to the flaring corona), and the capability of measuring magnetic fields through spectropolarimetry at visible and

University of Cambridge, UK

S. Fineschi

INAF - Osservatorio Astronimico di Torino, 20 Strada Osservatorio, Pino Torinese, Italy

A. Fludra

STFC Rutherford Appleton laboratory, UK

P.T. Gallagher

School of Physics, Trinity College Dublin, Dublin 2, Ireland

S. Imada, T. Shimizu

Institute of Space and Astronautical Science, JAXA, 3-1-1 Yoshinodai, Chuo-ku, Sagamihara, Kanagawa 252-5210, Japan

B. Kliem

Institute of Physics and Astronomy, University of Potsdam, Karl-Liebknecht-Str. 24-25, Potsdam 14476, Germany

V. Martínez-Pillet, H. Socas-Navarro, J. Trujillo-Bueno

Instituto de Astrofísica de Canarias, 38205 La Laguna, Tenerife, Spain

S. Parenti

Royal Observatory of Belgium, Belgium

S. Patsourakos

University of Ioannina, Department of Physics-Astrogeophysics Section, GR 45110 Ioannina, Greece

L. Poletto

CNR - Institute of Photonics and Nanotechnologies, Padua, Italy

R. Rutten

Sterrekundig Instituut Utrecht, The Netherlands

M. Siemer

DLR - Institute of Space Systems, Bremen, Germany

D. Spadaro

INAF - Osservatorio Astrofisico di Catania, Via S. Sofia 78, 95123 Catania, Italy

S. Tsuneta

National Astronomical Observatory of Japan, 2-21-1 Osawa, Mitaka, Tokyo 181-8588, Japan

R. Walsh

University of Central Lancashire, UK

P. Young

George Mason University, USA 
near-infrared wavelengths. Simultaneous spectroscopic measurements sampling the entire temperature range are particularly important.

These requirements are fulfilled by the Japanese Solar-C mission (Plan B), composed of a spacecraft in a geosynchronous orbit with a payload providing a significant improvement of imaging and spectropolarimetric capabilities in the UV, visible, and near-infrared with respect to what is available today and foreseen in the near future.

The Large European Module for solar Ultraviolet Research (LEMUR), described in this paper, is a large VUV telescope feeding a scientific payload of high-resolution imaging spectrographs and cameras. LEMUR consists of two major components: a VUV solar telescope with a 30-cm diameter mirror and a focal length of $3.6 \mathrm{~m}$, and a focal-plane package composed of VUV spectrometers covering six carefully chosen wavelength ranges between $170 \AA$ and $1270 \AA$. The LEMUR slit covers $280^{\prime \prime}$ on the Sun with $0.14^{\prime \prime}$ per pixel sampling. In addition, LEMUR is capable of measuring mass flows velocities (line shifts) down to $2 \mathrm{~km} \mathrm{~s}^{-1}$ or better.

LEMUR has been proposed to ESA as the European contribution to the Solar C mission.

Keywords Sun: atmosphere - Space vehicles: instruments - Techniques: spectroscopy $\cdot$ ESA Cosmic Vision

\section{Introduction}

For life on Earth, the Sun is the most important astrophysical object in the universe, and it holds a special place in the human imagination. Solar eclipses and the elusive appearance of the ghostlike corona have been a source of fascination since prehistoric times. Unfortunately, the impossibility of in-situ measurements at or near the solar surface and the complexity of the solar atmosphere make it difficult to study. Solar observations during the past several decades have yielded a wealth of new information and deep insights into the physical processes that occur in the solar atmosphere. Yet many questions remain unresolved. These include the heating of the solar chromosphere, the layer above the solar surface in which the temperature starts to increase with height, and the million degree corona (compared with the 6000 degree solar surface), the onset of coronal mass ejections - huge clouds of hot gas flung from the Sun at speeds of several $1000 \mathrm{~km} \mathrm{~s}^{-1}$ - and the origin of the solar wind, a constant outflow of coronal gas that fills the heliosphere and extends the solar atmosphere out to $100 \mathrm{AU}$ or more. Since these processes generate and simultaneously perturb the solar heliosphere, their understanding is central for answering the Cosmic Vision question: How does the solar system work? Moreover, the solar wind carries turbulent magnetic field out to the edge of the solar system where it drastically reduces the flux of incoming cosmic rays. These high-energy particles are harmful to life forms, especially highly evolved life forms. Understanding the solar magnetic system, its variability and severe modifications in large solar eruptions is therefore pivotal to answering the Cosmic Vision question: What are the conditions for planet formation and the emergence of life? It is particularly relevant to the sub-topic Life and habitability in the solar system. 
In almost all cases the primary impediment to progress in answering these questions is our inability to resolve and follow the small-scale structures produced by the processes that energize the solar atmosphere. Since these structures are intimately connected with the solar magnetic field, it is largely our inability to track the magnetic field from the photosphere into the corona that impedes progress. Many of the physical processes that produce activity in the solar atmosphere involve the creation, structuring, and dissipation of magnetic fields in a plasma, fundamental processes in all of astrophysics. Only on the Sun, however, do we have the possibility at least in principle to resolve the scales over which these processes take place, which makes studying them on the Sun of particular relevance for astrophysics as a whole.

Section 2 describes the scientific goals of the Solar C mission and the payload devised to achieve them. The specific scientific motivations of LEMUR and the derived technical requirements are discussed in Section 3. The mission profile is briefly discussed in Section 4 while a detailed technical description of LEMUR is given in Section 5. Finally, Section 6 summarizes the paper.

\section{Scientific Motivation for the Japanese Solar-C Mission}

During the last two decades, the availability of VUV (vacuum ultraviolet: 150 to $2000 \AA$ ) and X-ray telescopes and spectrometers with ever-increasing capabilities have greatly enriched our knowledge of the outer solar atmosphere. The Yohkoh mission provided detailed observations of coronal loops, jets, coronal arcade formation, and long-duration cusp-shaped flares. The SOHO mission facilitated a solar global view of solar transition region dynamics and structure, and made a giant leap in our observations and understanding of coronal mass ejections. Hinode data are providing new knowledge on photospheric and chromospheric dynamics and energy transport mechanisms, detailed temperature and density structures of active regions and their related dynamics, and are allowing tests of active region heating models such as the nanoflare model. However, key problems remain still unsolved. They include determining what role magnetic reconnection plays in producing solar flares (impulsive events that produce intense extreme-ultraviolet, X-ray, and sometimes gamma radiation) and coronal mass ejections, how particles are accelerated in the solar atmosphere, how the solar wind is energized and accelerated, and how small-scale sites of energy release determine the large-scale structures of solar activity.

During the past year solar physicists in Japan, the United States, and Europe have been studying the possibility of a follow-on mission to the highly successful Solar-A (Yohkoh) and Solar-B (Hinode) programs that would make major steps forward in improving our understanding of the solar atmosphere.

Solar-C will build on questions raised by but not answered by Hinode, in particular those regarding the dynamical coupling between the various components of the solar atmosphere, considered as an "integrated system". Indeed, until now, solar physics space research has mainly concentrated on selected regions of the solar atmosphere, such as the corona or photosphere, and considerable progress has been made in observing structures and dynamics in these regions. Nevertheless, important Hinode results such as the discoveries of chromospheric microjets and "type-II" spicules 
suggest that what is required is the capability of measuring energy release over the entire range of spatial, spectral, and temporal scales observed in different atmospheric regions. These scales often differ markedly in the chromosphere, transition region, and corona. Currently, even the combination of data from several existing missions do not produce adequate scale coverage. Combinations of data from different missions also tend to be restricted by difficulties in data co-registration and simultaneity.

The unifying concept behind Solar-C is thus the need to understand how the solar magnetic field drives the flow of mass and energy from the thermally dominated lower solar atmosphere into the magnetically dominated corona and solar wind acceleration region as a coupled system. Understanding how this flow takes place requires detailed knowledge of the magnetic field not only in the photosphere but also above the $\beta=1$ surface (where $\beta$ is the kinetic gas to magnetic pressure ratio), in the chromosphere. Here, the proposed instrumentation for Solar- $\mathrm{C}$ will make revolutionary new measurements of the magnetic field with far better stability, polarimetric accuracy and temporal coverage than is possible from ground-based telescopes [e.g., 1], Besides providing accurate and proper boundary conditions for extrapolating the field into the corona, knowledge of the chromospheric field also allows tracing the release of free energy of the magnetic field and its effects on the momentum and energy balance of the chromosphere and corona. Since the forcing of the field implies that much of the magnetic energy release occurs well above the photosphere, such studies are almost impossible using only photospheric fields. This, along with all-encompassing plasma diagnostic capabilities and with the highest resolution solar observations ever made at all temperatures present throughout the atmosphere, will produce fundamental advances in our understanding of how field and plasma processes form structures, how magnetic energy is released through instabilities, reconnection, wave dissipation, and particle acceleration, and what role the magnetic fields play in heating and accelerating plasma in the corona and the solar wind. Solar-C will be the first mission to reveal the spatial and temporal scales over which energy release in the solar atmosphere is aggregated into observable structures.

Solar-C will answer to the following key questions, that form the mission science goals:

1. How are elementary atmospheric structures created and how do they evolve in each temperature domain of the atmosphere?

2. How is energy transported through small elementary structures into the largescale corona and how does it drive the solar wind?

3. How is magnetic energy dissipated in astrophysical plasmas?

4. How do small-scale physical processes initiate large-scale dynamic phenomena creating space weather?

These key unresolved science questions can only be addressed with an instrument suite with broad temperature coverage so that emission from the photosphere, chromosphere, transition region, and corona can be observed simultaneously. Another critical requirement for this mission is high resolution. High spatial resolution is necessary to reveal how the filamentary magnetic field in the photosphere is relaxed from a forced to an almost force-free state at chromospheric heights. High temporal resolution is necessary to capture the dynamics of the chromosphere and transition region 
as they connect to the corona, and high spectral resolution is required to provide diagnostics of the magnetic field and energy flow through detailed measurements of line profiles. These observational requirements can be met by a spacecraft placed in a geosynchronous orbit with a payload of three state-of-the-art instruments:

- a Solar UltraViolet, Visible, and Infrared Telescope (SUVIT);

- an X-ray or extreme-ultraviolet imaging telescope (XIT);

- a vacuum ultraviolet ( $150 \AA \AA$ to $2000 \AA$ ) high-throughput spectroscopic telescope (proposed here with acronym LEMUR).

These instruments and their science cases have been studied in detail by the JAXA Solar-C International Working Group, with significant contributions by scientists from European countries and the US. Detailed descriptions are given in the Japanese Draft Interim Report ${ }^{\mathrm{i}}$. The basic characteristics of each instrument are summarized in Table 1.

Table 1 Solar-C instrumentation payload summary

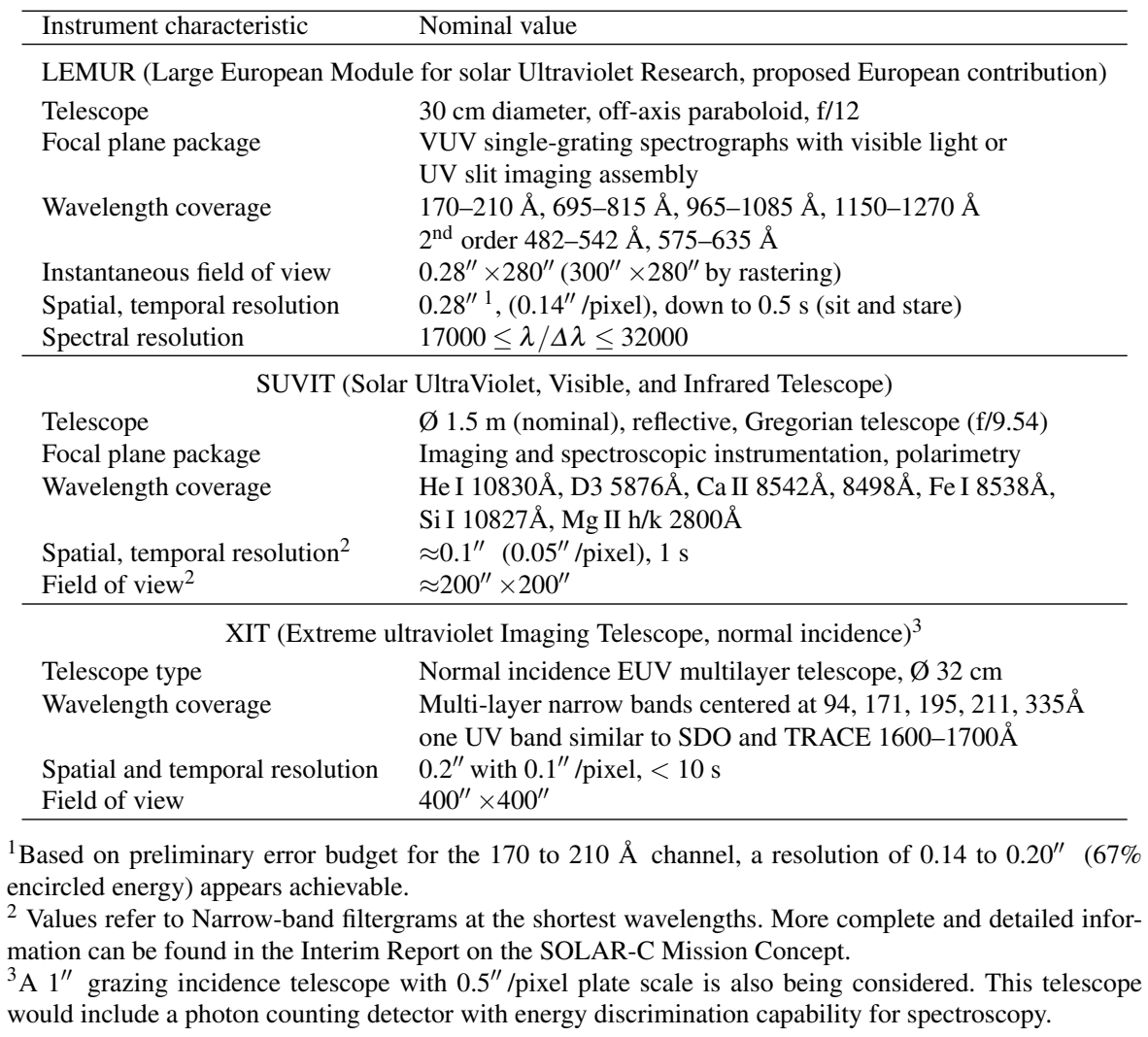

i SOLAR-C Working Group, 2011, Interim Report on the SOLAR-C Mission Concept. http://hinode.nao.ac.jp/SOLAR-C/archive_e.html 


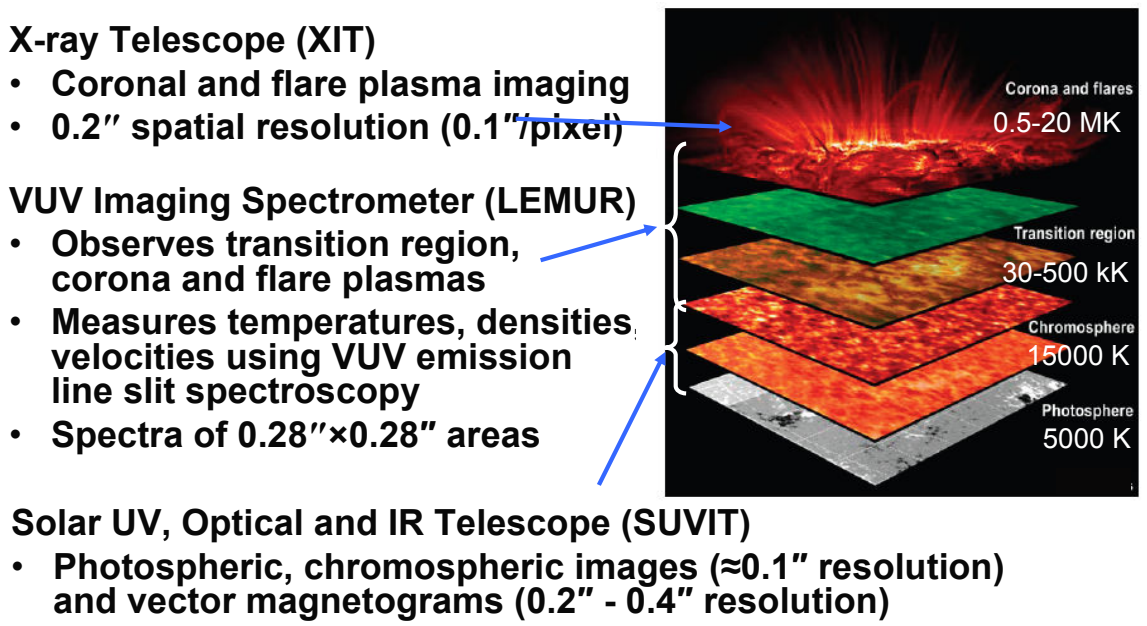

Fig. 1 The Solar-C observation space. All regions of the solar atmosphere are observed with simultaneous and matched high spatial and spectral resolution. All figures are in color in the online version.

These instruments will provide overlapping temperature coverage and complementary capabilities such as spectroscopy and narrow wavelength band imaging, high cadence and throughput, and will also feature active region sized fields-of-view. A pictorial representation of the overall Solar-C observational domain is shown in Figure 1.

Solar-C will be highly synergistic with, and complementary to, the forthcoming Solar Orbiter and Solar Probe+ missions ${ }^{\mathrm{ii}}$ of ESA and NASA. Besides stereoscopic observations with Solar Orbiter, in-situ composition measurements made from Orbiter and Probe+ will be traced to their solar origin by Solar-C observations. Solar-C will greatly extend the important new information on the chromosphere and transition region that will be provided by the up-coming NASA IRIS mission. In fact, the latter does not have adequate transition region and coronal coverage, does not measure chromospheric magnetic fields, and will observe the chromosphere with a lower spatial resolution than the Solar-C optical telescope. Finally, Solar-C will also occur at a time when major ground facilities such as the European Solar Telescope (EST) and the Advanced Technology Solar Telescope (ATST) will become operational, allowing highly complementary observations such as a direct measurement of the coronal magnetic field at the limb.

Solar- $\mathrm{C}$ will have the ability to determine what energizes and maintains the plasma in the complex magnetic environment of the solar atmosphere. It will be able to measure the signatures of all major processes that form structures and release energy

ii currently foreseen to be launched in 2017 and no later than 2018, respectively 
such as magnetic reconnection and wave dissipation. Theoretical work tells us that the smallest spatial scales over which important physical processes such as magnetic reconnection, wave dissipation, and particle acceleration occur may be only meters in size, far smaller than will be resolvable with solar telescopes for the foreseeable future. Solar observations, however, suggest that these physical processes are coherent over much larger spatial scales. Chromospheric jets observed with Hinode, for example, have spatial scales of about $0.2^{\prime \prime}$ to $0.3^{\prime \prime}$ (about 140 to $210 \mathrm{~km}$ at the Sun). Similarly, observations of transition region and coronal structures taken with $>1$ " resolution instruments indicate that at least $10 \%$ of the observed area is filled with emitting plasma, again suggesting structures with spatial scales that can be observed by the next generation of solar instruments.

It is widely believed that magnetic reconnection plays a central role in many solar phenomena, such as flux emergence and cancellation in the photosphere, chromospheric and coronal jets, and coronal eruptions (flares and coronal mass ejections). The physics of magnetic reconnection, however, is still poorly understood. To further progress, the plasma conditions in and around the current sheet must be explored in detail. This motivates the selection of multiple wavelength ranges so that the brightest emission lines at critical temperatures can be observed spectroscopically with high spatial resolution. Another aim for Solar-C is to observe the signature of reconnection in the lower atmosphere in order to explore this process in partially ionized plasma, which is highly relevant in many astrophysical settings, for example in starforming regions. This can also shed light on the surprisingly short time scales of chromospheric energy release events discovered by Hinode and can directly address the mechanisms of flux emergence and cancellation.

The primary science goals of the high resolution Solar-C mission, understanding the magnetic field throughout the solar atmosphere and the release of energy through magnetic reconnection, wave dissipation, and particle acceleration, lay the foundation for studying a wide range of complex solar phenomena. Of particular interest are phenomena that influence the near-Earth environment, such as coronal mass ejections (CMEs), flares, and the solar wind. Predicting the occurrence of CMEs and flares requires understanding the storage and release of magnetic energy in the solar atmosphere. It is clear that these occur on very different time scales. Free magnetic energy is built up slowly through the emergence, shearing, and cancellation of flux in the photosphere. The release of energy, in contrast, occurs rapidly, but what actually triggers an eruption has not been firmly established.

The solar wind is another important component of space weather. Coronal holes are the source of the fast solar wind (700 to $800 \mathrm{~km} \mathrm{~s}^{-1}$ at Earth orbit). The origin of the slow solar wind ( 300 to $400 \mathrm{~km} \mathrm{~s}^{-1}$ ) is unknown. Recently, instruments on Hinode have detected persistent high speed outflows from large areas at the periphery of many active regions [e.g., 2]. These outflows are characterized by bulk shifts in the line profile of up to $50 \mathrm{~km} \mathrm{~s}^{-1}$ and enhancements in the blue wing of up to $200 \mathrm{~km}$ $\mathrm{s}^{-1}$ [e.g., 3]. These outflows are of interest because they may lie on open field lines and contribute to the solar wind [4].

Despite the extensive work on the outflows that has been done with Hinode a number of important questions remain. Perhaps the most important solar wind related question that Solar-C can address is how these outflows are heated and accelerated. 
Temporally resolved observations suggest that the outflows are composed of episodic events that originate low in the solar atmosphere. Solar- $\mathrm{C}$ will be better able to study these outflows than other missions because of its wide temperature coverage. Solar-C can determine with precision at what temperatures the outflows begin in the atmosphere and reveal, with its high spatial resolution, the magnetic structuring at those temperatures, providing the observations necessary to understand how these outflows are formed and connect to the heliosphere as part of the slow solar wind.

\section{LEMUR Scientific Goals and Requirements}

LEMUR provides diagnostic capabilities that are essential for achieving the Solar-C science goals. It consists of a large VUV telescope feeding a set of spectrometers and cameras capable of observing simultaneously with high throughput all temperature ranges ( 0.01 to about $20 \mathrm{MK}$ ) of the outer atmosphere with: (a) high spatial sampling (about $200 \times 200 \mathrm{~km}^{2}$, and down to $100 \times 100 \mathrm{~km}^{2}$ in the 170 to $210 \AA \mathrm{A}$ band), (b) high spectral resolution (down to $2 \mathrm{~km} \mathrm{~s}^{-1}$ by line centroiding, ensuring dynamical scale coverage), (c) plasma diagnostic tools (such as electron density measurements), and (d) high time resolution (down to $10 \mathrm{~s}$ for raster scans of a $4000 \mathrm{~km}$ wide region with $200 \mathrm{~km}$ sampling).

Moreover, LEMUR optics are designed to achieve the low scattering performance necessary to observe, for example, the full life span of transient events, and to explore faint reconnection outflow regions, coronal holes, and far off-limb observations up to 0.5 solar radii above the limb. Finally, LEMUR is able to determine absolute coronal velocities using wavelengths of chromospheric lines as zero-velocity references.

The solar chromosphere/transition region, with temperatures between $0.02 \mathrm{MK}$ (upper chromosphere) and 1 MK (corona), strongly emits in the VUV. Strong lines from increasingly higher ionization stages generally fall at shorter wavelengths. The chromosphere and lower transition region up to about $0.2 \mathrm{MK}$ can be observed at wavelengths $\geq 900 \AA$ while the upper transition region between about $0.2 \mathrm{MK}$ and $1 \mathrm{MK}$ and the corona $\geq 1 \mathrm{MK}$ are best observed below $900 \AA$. These relationships of temperatures to wavelengths allow us to select wavelength ranges for LEMUR that maximize the range of temperature sensitivity. For the first time, the full characterization of the entire transition region and corona, and their linkages to the chromosphere, will be achieved by a single instrument. Moreover, a slit imaging assembly ensures that the LEMUR slit spectra have excellent spatial co-alignment with the photospheric and chromospheric magnetograms and filtergrams provided by SUVIT at even higher resolution, ensuring that LEMUR observations are always understood in the context of the surrounding lower atmosphere.

VUV spectroscopy has several fundamental advantages over imaging, such as provided by the AIA instrument aboard SDO. First, it can provide direct measurements of electron temperatures, densities, and chemical abundances by using line ratios, which, together with line profile/Doppler-shift measurements, provide fundamental inputs to any theoretical modeling. Second, imaging instruments mostly show denser, cooling plasmas and provide very limited information on the plasma that is being heated, and on plasma velocities. Moreover, the information on tem- 
perature and plasma velocity provided by spectroscopy improves the effective spatial resolution in two ways. One is the ability to see throughout the atmosphere at different specific temperatures. The solar corona is largely optically thin so multi-thermal structures can often not be resolved along the line-of-sight in broadband imaging data due to the presence of additional spectral lines formed at different temperatures just within a few Angstroms of the EUV spectrum [5]. The other is the ability to separate the emission in velocity space, i.e., to measure plasma characteristics such as densities along the line-of- sight for plasma flowing at different velocities.

Current and forthcoming VUV imaging spectrometers such as CDS, SUMER, and UVCS on SOHO, EIS on Hinode, and SPICE aboard Solar Orbiter either acquire simultaneously a few fixed restricted regions of the VUV spectrum or access a large spectral range by scanning in wavelength and therefore, unlike LEMUR, have limited instantaneous temperature coverage. The required spectroscopy needs high sensitivity and large telemetry volume, beyond what has been available with past and current satellites, but that will be provided by Solar-C.

\subsection{Impulsive energy release throughout the solar atmosphere (Goals 1 and 3)}

Energy release is frequently highly dynamic, involving turbulent motions and mass flows. The magnitudes of the flows and turbulent motions have complex temperature dependences, and the most dynamic motions do not always occur at the highest temperatures. The chromosphere and lower transition region are far more dynamic than the quiet corona. Waves, turbulent motions, and small eruptive events are common in the chromosphere and transition region.

For example, possible heating via reconnection in the transition region is suggested by observations of explosive events with plasma flow velocities close to the Alfvén speed $\left(100 \mathrm{~km} \mathrm{~s}^{-1}\right)$, sometimes seen as bi-directional jets, in high resolution UV spectra of transition region lines [6]. These explosive events are in contrast to an apparent steady average downflow motion in the transition region of $\approx 2$ to $25 \mathrm{~km}$ $\mathrm{s}^{-1}$ and non-thermal motions of the order of $20 \mathrm{~km} \mathrm{~s}^{-1}$ [e.g., 7].

The signatures of energy transport and release from solar phenomena never occur at just one temperature in the atmosphere. For example, in a solar flare radiation is emitted across the electromagnetic spectrum from plasmas that span a temperature range from about $0.01 \mathrm{MK}$ to about $40 \mathrm{MK}$. There is in addition a non-thermal component that produces $\mathrm{MeV}$ particles sufficiently energetic to produce nuclear reactions in the atmosphere. As more quiescent examples, magnetic loops in active regions span temperature ranges from about $0.01 \mathrm{MK}$ to $3-4 \mathrm{MK}$, and most spicules have temperatures significantly less than $0.01 \mathrm{MK}$. Every dynamical event exhibits related multi-thermal phenomena. This is true also for the diffuse solar corona. Thus, diagnosing multi-thermal plasmas is required to understand energy release in the solar atmosphere.

Waves are important throughout the solar atmosphere as a diagnostic tool for determining the conditions in the plasma they are propagating in. The rapidly-developing field of chromospheric/coronal seismology aims to combine the magnetohydrodynamic theory of waves in this structured environment with emission line intensity, 


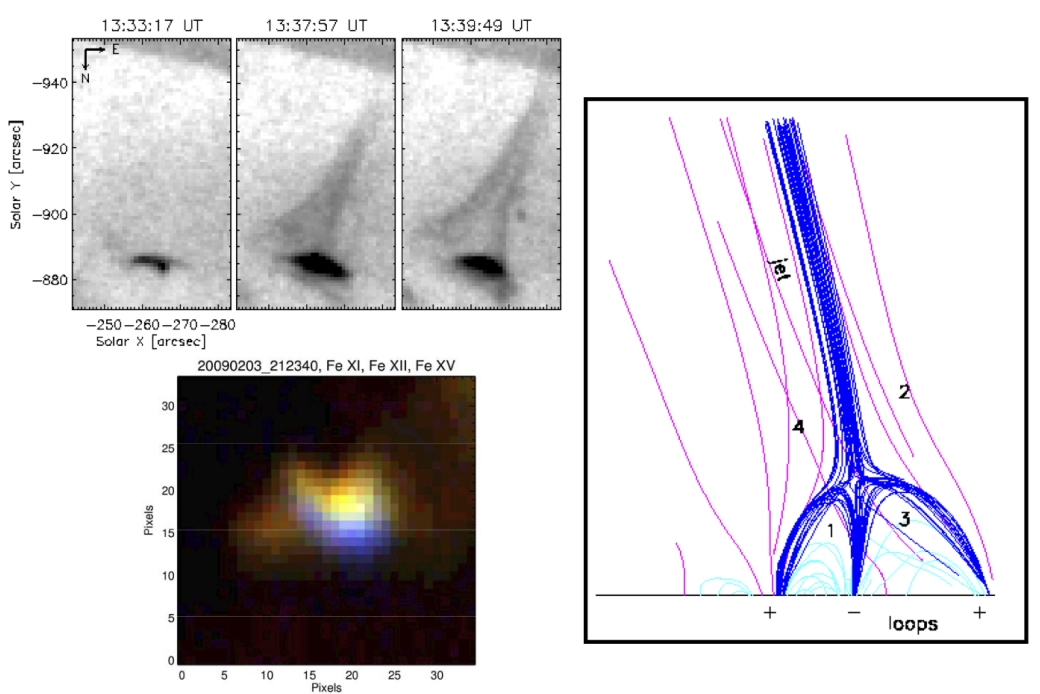

Fig. 2 Top left: Hinode/XRT images of a jet from a bright point. Lower left: Hinode/EIS composite image of a bright point. The color code is red (Fe XI, 1.4 MK), green (Fe XII, 1.6 MK), and blue (Fe XV, $2 \mathrm{MK}$ ). The bright point looks like a small loop, brightest at the top. The separation of the two footpoints is only $5800 \mathrm{~km}$. The EIS pixel size is $1^{\prime \prime}$, so it is clear that the loop is not at all well-resolved. Right: A theoretical schematic of the magnetic configuration of a bright point region and a jet.

density, and Doppler-shift measurements to determine detailed physical parameters in the corona such as the magnetic field, the density stratification, and heating and energy dissipation processes. Moreover, since waves and oscillations are often initiated by impulsive heating processes, their observation provides an additional window into how the corona is heated. Both kink-mode and longitudinal slow mode standing and propagating waves have been detected in the corona [e.g., 8; 9], and EIS on Hinode has been able to detect small amplitude waves [e.g., 10]. But, because of the limitations of current instrumentation, their diagnostic potential has not yet been fully realized. Doing this requires spatially-resolved measurements at high time cadence in emission lines formed over a range of temperatures along the basic structural elements of the corona. The study of oscillation and wave phenomena at all temperatures by LEMUR via seismological techniques will allow us to determine poorly-known but crucial dissipation coefficients at sub-arcsec scales for the first time.

Coronal hole bright points and their associated jets are an example of energy release over a small localized area. They appear to be the result of magnetic reconnection when an erupting bipole encounters existing open magnetic flux. The simplicity of the bright points and their isolation from other heating events makes them attractive targets for reconnection studies. Figure 2 shows XRT and EIS images of a jet and a bright point, respectively, and the schematic of a reconnection model. Hinode observations have shown that the heating and cooling typically occurred on timescales 
of tens of seconds, too short for the imaging capabilities of EIS, but easily achievable with LEMUR. The spatial scales of jets should be resolved by LEMUR. The plasma conditions in the jets are crucial for testing reconnection models.

LEMUR can determine the topology and morphology of the magnetically dominated structures $(\beta<1)$ as well as the thermodynamic state of the plasma (its motions, density, temperature, turbulence, and abundances) that fills these structures. This provides the required input for the computation of the large-scale coronal field by next-generation extrapolation techniques, which will include non-magnetic forces [11] and plasma flows.

\subsection{Ubiquitous heating processes of the solar atmosphere (Goal 2)}

The source of the hot and tenuous plasma making up the solar corona is still debated. The traditional view is that heating occurs high in the corona, probably in an impulsive fashion by reconnection events or by dissipation of steepened wave packets. This generates a downward thermal heat flux which heats and evaporates chromospheric plasma into the corona. Transverse magnetic waves can carry enormous amounts of energy upward through the chromosphere [12; 13]. However, Solar-C's high resolution and spectroscopic capability at a wide range of temperatures is required to trace the propagation, mode-coupling, reflection, and dissipation of these waves as they cross the $\beta=1$ surface into the transition region and corona. Critical is the ability to resolve their short periods $(<60 \mathrm{~s})$ and small amplitudes and velocities $\left(<1^{\prime \prime}\right.$ and $<20 \mathrm{~km} \mathrm{~s}^{-1}$ ).

An alternative view is that a subset of chromospheric spicules can heat up to coronal temperatures as they rise and supply mass to the corona. These "type II" spicules have typical widths of $\approx 0.3^{\prime \prime}$, upflow speeds of 50 to $100 \mathrm{~km} \mathrm{~s}^{-1}$ [14], and generate observable features (e.g., asymmetries) in the line profiles of several coronal spectral lines as observed at 2 " spatial resolution by EIS on Hinode. These findings have been recently substantiated by SDO/AIA observations [15]. However, spectroscopic observations at comparable spatial resolution are necessary to establish whether mass is effectively supplied to the corona.

The problems encountered in understanding the coupling between the chromosphere and the corona are illustrated in Figure 3. The chromosphere has relatively large patches $\left(10^{\prime \prime}\right.$ to $\left.20^{\prime \prime}\right)$ containing many thin, bright spicules, and the coronal structures are narrow, isolated loops that fade at their footpoints. There is little correspondence between the two. A comparable transition region image which can only be obtained by a rastering spectrometer would take almost two hours to complete with SUMER. Although the large-scale structures do not normally change on this timescale, the small-scale dynamics are much faster. LEMUR, having more than 30 times higher throughput than SUMER in transition region lines, will make a giant step forward.

In particular, LEMUR can connect in a systematic manner photospheric phenomena with coronal phenomena because the chromosphere/transition region interface will be observed with matching resolutions both below (chromosphere and photosphere) and above (corona). The complementary magnetic maps provided by the vis- 


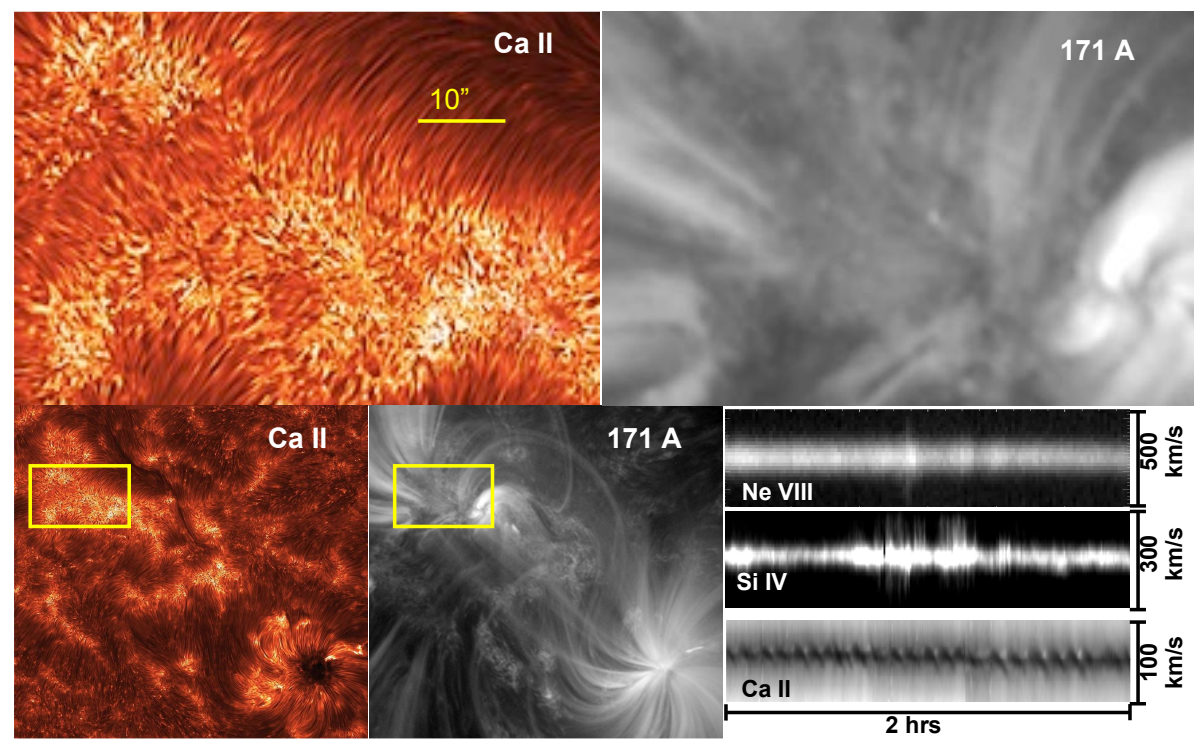

Fig. 3 The chromosphere-corona connection. The top panels show cut-outs taken from the chromosphere (Ca II, resolution similar to LEMUR) and the lower corona (171 $\AA, \sim 1^{\prime \prime}$ resolution) images. The regions are outlined in yellow. The bottom right panel shows representative time series of chromospheric (Ca II), transition region (Si IV), and lower coronal (Ne VIII) line profiles. The chromospheric spectra have characteristic 3 to 5 min periods, the transition region brightenings occur in bursts of up to $30 \mathrm{~min}$, while coronal variations are more diffuse.

ible telescope on Solar-C assures that the LEMUR observations can always be traced back to the plasma and field conditions in the lower atmosphere.

SOHO/CDS observations [e.g., 16] have shown that active region emission between 2 and $3 \mathrm{MK}$ is dominated by diffuse emission, i.e., unresolved at the current best $\left(1^{\prime \prime}\right)$ resolution. However, at lower temperatures, active regions contain 'warm' loops that reach temperatures no greater than about 1.4 MK and appear not very far from being resolved. This is seen in 1" EUV imaging (TRACE, SDO/AIA), and by measuring densities and emission measures (hence path lengths and spectroscopic filling factors) with EUV coronal spectrometers (CDS, EIS). Indeed the footpoints of these warm loops are anchored in magnetic field concentrations of a few arcsec in size. At higher temperatures, in their core, active regions have hot (3 to $4 \mathrm{MK}$ ) loops, frequently unresolved in imaging data.

Thus, current observations of the corona indicate the need for higher spatial resolution data. LEMUR's spatial resolution should completely resolve at least the warm loops. LEMUR can provide the same high spatial resolution throughout the entire solar atmosphere and provide by far the broadest diagnostics to measure the physical conditions of loops.

The fact that we resolve observationally a structure does not mean that it could not be composed of unresolved magnetic threads. Indeed this is one of the assumptions about nanoflare heating of coronal loops. The plasma confined to these threads must 
be heated sequentially in order to explain the longevity and densities of these loops [17]. One issue is whether plasma is heated in a steady or impulsive way. Detailed measurements from Hinode/EIS are shedding some light into these issues; however it still takes a very long time (tens of minutes) to scan an active region loop from footpoint to footpoint. The much higher sensitivity and telemetry of LEMUR will allow us to determine the relative roles of steady and impulsive heating in active region structures and the validity of models such as the nanoflare model.

\subsection{Solar flares (Goal 3)}

Solar flares form the high-energy tail of reconnection events. They are therefore ideal for studying how magnetic energy is dissipated via reconnection, one of the important goals of the Solar-C mission.

Figure 4a presents a sketch of the standard flare scenario that successfully explains features seen in X-ray and EUV images, such as flare arcades, ribbons, plasmoid ejections, supra-arcade downflows, and hard X-ray loop-top emission. The predicted inflows, shocks and high-velocity jets, are central to this scenario but they must be faint because they have not been seen in images. If they exist, they should stand out as Doppler shifted features in LEMUR spectra. Also, rapid heating at the shocks might result in non-equilibrium ionization and different ion and electron temperatures which can be investigated with high cadence spectra. An example of the non-equilibrium ionization structure of slow-mode shocks is shown in Figure $4 \mathrm{~b}$. The temperature evolution in the shocks is tracked by measuring the intensities of lines of highly ionized iron from Fe XVII through Fe XXIV.

The right part of Figure $4 \mathrm{c}$ is an Fe XXI spectral image taken with SUMER, showing high velocity (400 to $1000 \mathrm{~km} \mathrm{~s}^{-1}$ ), high temperature (10 MK) plasma at many positions along the spectrometer slit, above a flare arcade. These observations are highly suggestive of dynamics in or close to the reconnection region, but SUMER was observing in sit and stare mode and the time cadence was not better than 2 minutes (due to limited telemetry), so the structure and dynamics could not be resolved. Moreover, SUMER flare observations were restricted to the off-limb because of its detector characteristics. Fast, high resolution Doppler mapping in the full complement of hot Fe lines (Fe XII to XXIV) within and above the forming X-ray loops, together with high cadence X-ray or EUV imaging observations, such as the context TRACE image shown in Figure 4, are required to determine the current sheet geometry and to test the standard flare scenario.

More in general, it is clear that magnetic reconnection in the corona occurs much faster than the time scales predicted by the classic Sweet-Parker model, but what drives this fast reconnection? Recent theoretical work has suggested that it is the formation of magnetic islands in the current sheet that leads to fast reconnection (e.g., $[18 ; 19])$. To reconcile the observations with theory and numerical models, the physical conditions in and around the current sheet must be explored in detail. Of particular importance are the inflow and outflow velocities. Since the density in this region is close to that of the ambient corona and the volume is small, such measurements are very challenging. This further motivates the selection of multiple wavelength ranges 


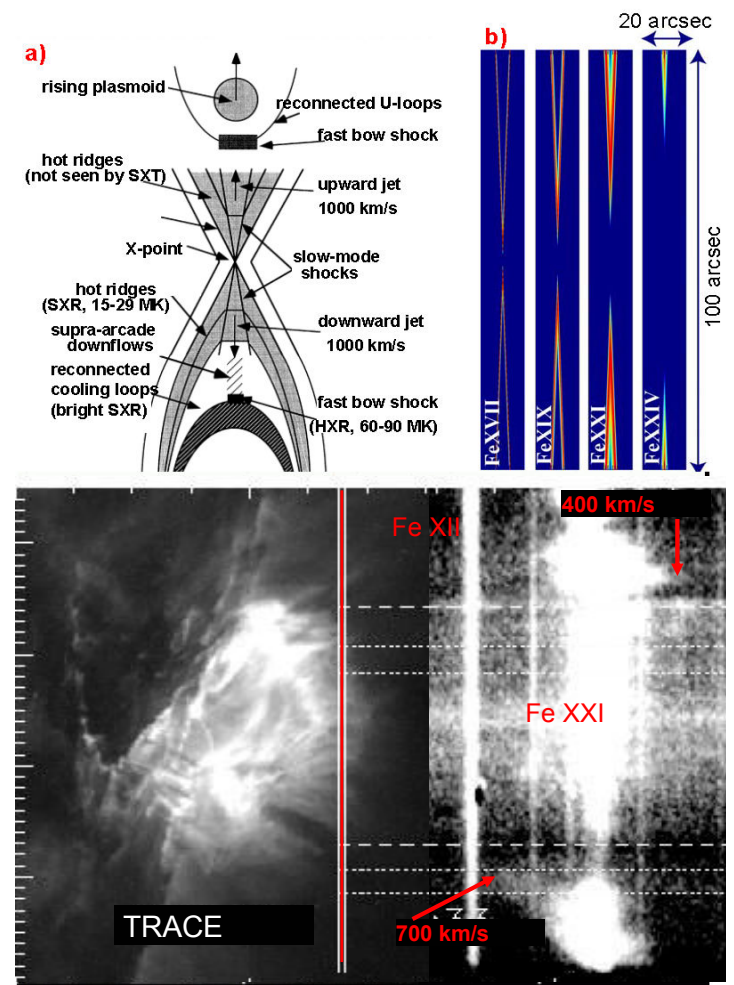

Fig. 4 The standard flare model. a) cartoon showing the relationship between reconnection jets, shocks, and the flare arcade. b) computation of non-equilibrium ionization structure in $1200 \mathrm{~km} \mathrm{~s}^{-1}$ jets. c) large flare observed by TRACE and SUMER. The position of the SUMER slit is shown as a red vertical line on the TRACE image.

so that the brightest emission lines at critical temperatures can be observed spectroscopically with high spatial resolution.

\subsection{Large-scale dynamic phenomena (Goal 4)}

CMEs and flares are closely related phenomena which begin near small sections of a photospheric polarity inversion line (Figures 4 and 5). Their onset is typically associated with the emergence of new flux or with the dispersion and cancellation of old flux, generally with a high degree of fragmentation into the smallest observable scales. Recent observational and computational studies indicate that a topology change from a magnetic arcade to a flux rope occurs in either case, before or in the early stages of the eruption $[20 ; 21]$. Observing and quantifying this topology change is one of the greatest challenges in space weather research. It requires unraveling the signatures of reconnection at the smallest scales of the flux fragments and learning 
how these organize into larger scales to form a coherent magnetic structure of nearly active-region size that is able to erupt. In the corona, such signatures have been seen as small-scale brightenings and motions of brightened plasma along filaments and filament channels, but a comprehensive observation of the evolution from small to large scale has not yet been obtained. Moreover, on theoretical grounds, the reconnection is expected to occur also at photospheric and low chromospheric levels [22]. It may well be relevant also in the intermediate height range. The capability to observe line shifts and broadenings across a wide range of temperatures represents LEMUR's unique potential to discover signatures of reconnection through several layers of the atmosphere. The high spatial resolution of such measurements will allow deeper insights into the topology changes preceding CMEs and flares.

LEMUR's broad temperature coverage has the potential to discover links between cool filament plasma trapped in the gradually destabilizing flux and the ambient hot coronal structures (e.g., coronal cavities). Since the reconnection that forms the erupting flux is thought to often involve also the ambient flux, a linkage may frequently occur, possibly only transiently, and hold important clues to the genesis of the eruptions.

As soon as the eruptions are triggered, the unstable flux undergoes a rapid and huge expansion. Often the magnetic connections in the source regions are further changed, transient coronal holes are produced, and the corona is perturbed on a semiglobal scale, which may involve the triggering of eruptions at remote locations, i.e., very complex space weather effects. Combining LEMUR and large-scale EUV (or $\mathrm{X}$-ray) imaging observations allows following the evolution to the largest scales and mutually verifying and complementing the diagnostics, for example by correlating line shifts and broadenings with images of transient coronal holes. Only spectroscopic observations across a substantial temperature range allow fully detecting the
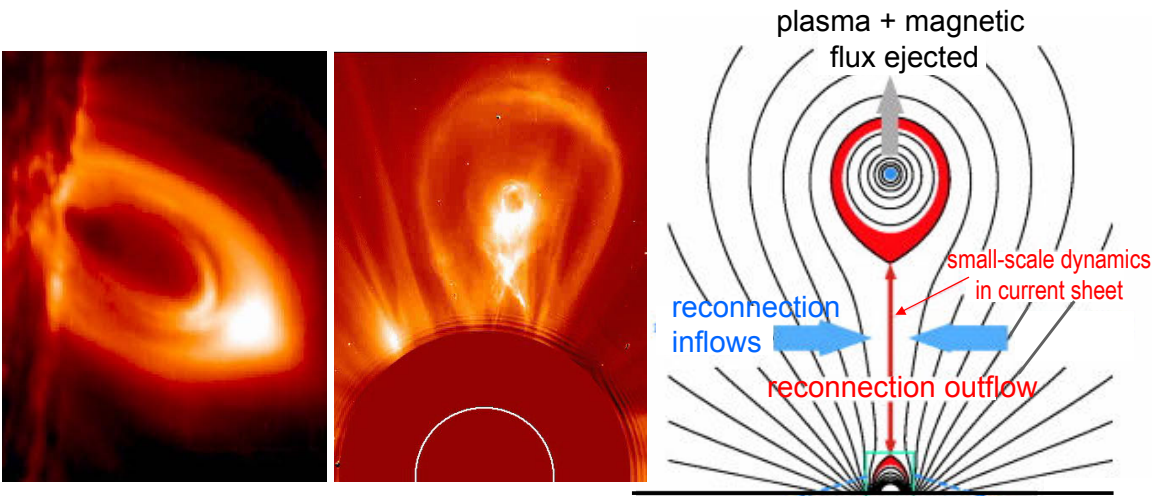

Photosphere

Fig. 5 Typical flare loops (EUV, left), coronal mass ejection (CME, white-light, center) and schematic CME model (right). 
plasma streaming into the legs of CMEs from the areas of transient coronal holes, thus completing the diagnostics of the mass and energy budget of the events [23].

\subsection{The solar wind (Goal 2)}

Despite many observations, it is unclear which structures and what physical mechanisms are primarily responsible for the acceleration of the slow and fast solar wind. Figure 6 summarizes the state of our understanding. LEMUR's capability of measuring the physical parameters of the coronal plasma at greater heights and with higher accuracy than previous missions, and in combination with the new near-Sun in-situ measurements of Solar Orbiter and Solar Probe+, will likely clarify the fundamental issues. For example, LEMUR is capable of measuring relative element abundances in different atmospheric structures that can be related to in-situ measurements. Element abundances in the slow solar wind are different from those in the fast solar wind, with the abundances of low-FIP (First Ionization Potential) elements enhanced compared to high-FIP elements. The abundances of the fast solar wind are close to the photospheric abundances. Thus, abundance measurements can be used in conjunction with in-situ measurements to locate the origins of the solar wind.

It is well known from the early comparisons of solar X-ray flux and in-situ measurements of particle speeds that coronal holes $(\mathrm{CH})$ are the source of the fast solar wind [e.g., 24]. For polar coronal holes, outflows of the order of 10 to $20 \mathrm{~km} \mathrm{~s}^{-1}$ have been found at the boundaries of magnetic network cells from SOHO/SUMER observations in the Ne VIII $770 \AA$ line formed at the base of the corona $(\mathrm{T}=0.63 \mathrm{MK})$. The flows, as shown in Figure 7a, appear stronger in areas of reduced Ne VIII emission [25]. From SUMER measurements of C IV and Ne VIII lines, respectively, the wind starts flowing between $5 \mathrm{Mm}$ and $20 \mathrm{Mm}$ and flows through magnetic funnels [26]. Despite this progress, there is still a substantial lack of knowledge as to which structures within coronal holes and what physical mechanisms are primarily responsible for the acceleration of the fast solar wind. Three candidates have been discussed as the structures responsible: plumes, interplumes, and jets (see Figure 6).

White light images of the solar minimum corona during eclipses reveal linear structures, called plumes, rooted in coronal holes and extending towards interplanetary space. These plumes could supply mass to the solar wind; measurements from SOHO/CDS have suggested that they have near photospheric abundances at their base [28]. Observations in VUV spectral lines formed at temperatures between 0.8 and $1 \mathrm{MK}$ allow the study of the plume roots where reconnection with newly emerging flux has been suggested to cause their formation.

Plumes are known to be denser and cooler than the surrounding interplume regions, however direct measurements with SUMER were limited, mainly by sensitivity. Spectral lines are observed to be broader in interplume regions, hinting at preferential energy deposition at these sites. SUMER observed smaller outflow speeds at the base of polar plumes, so interplume regions have been suspected to be the source regions of the fast wind. However, contradicting results have been obtained from SUMER observations based on the Doppler dimming technique applied to offlimb spectra [e.g., 29; 30]. LEMUR Doppler measurements with an accuracy of 


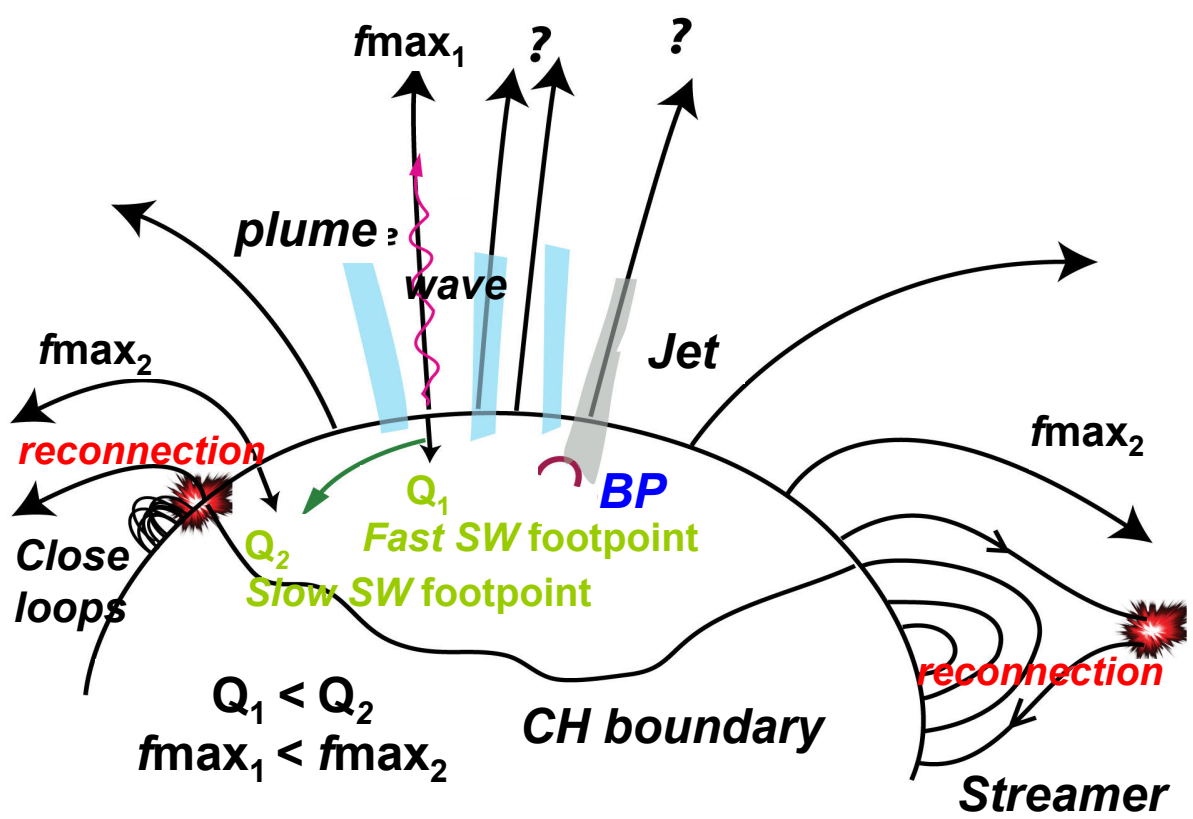

Fig. 6 A schematic showing various atmospheric structures found in coronal holes and possible sources of the fast and slow solar wind. The quantities fmax and Q refer to the rates of magnetic expansion and heating discussed for example by [27] and references therein.

$\leq 2 \mathrm{~km} \mathrm{~s}^{-1}$ in coronal spectral lines are needed, together with off-limb observations with the lowest possible level of stray light (disk radiation scattered by telescope optics). LEMUR can also greatly extend and improve direct temperature measurements by observing temperature sensitive line ratios (e.g., from Mg IX) as well as spectral lines separated considerably in wavelength, i.e., having substantially different excitation energies (e.g., from O VI and Fe XII). Moreover, comparing the chemical composition of plume and inter-plume regions in the corona from LEMUR spectra with in situ data by Solar Orbiter and Solar Probe+ at elevated latitudes could establish which structures are the primary sources of the fast solar wind.

Wave diagnostics are available for LEMUR as a tool for investigating the plasma properties of plumes and inter-plume regions. Quasi-periodic fluctuations in intensity have been observed in plumes and inter-plume regions, with periods of the order of 5 to $20 \mathrm{~min}$ [e.g., 31], and possibly are signatures of slow magnetoacoustic waves. However, accurate measurements of densities and temperatures (as well as Doppler shifts) at much higher signal to noise ratio and cadence (10 to $20 \mathrm{~s}$ timescales) than currently available in the low corona, as can be provided by LEMUR, are needed to confirm this scenario and to investigate higher frequencies.

The high spatial resolution of the Solar-C payload can provide a substantial increase in our understanding of the polar regions, even from $1 \mathrm{AU}$, on a path opened by the Hinode discoveries on the polar magnetic landscape [32]. LEMUR, in particular, is able to observe the poles with an effective (considering fore-shortening effects at an 


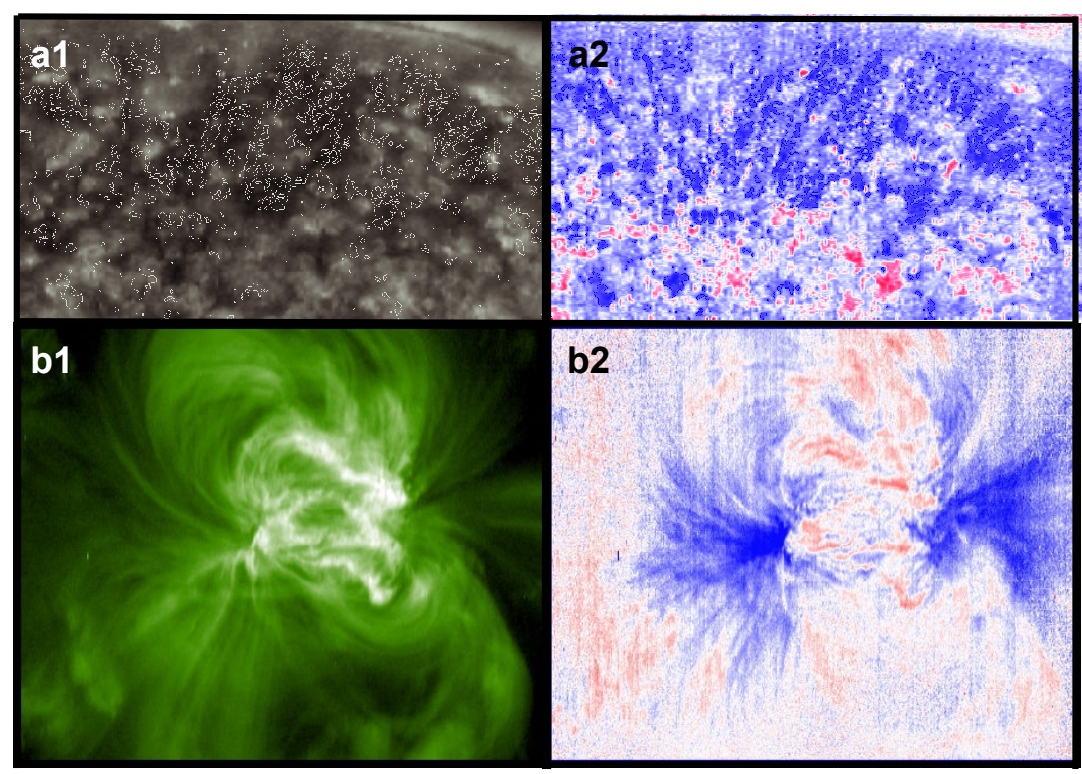

Fig. 7 Wind outflows at the base of the corona: Panels al and a2: A large portion of the north polar coronal hole imaged in the Ne VIII $770 \AA$ line with SUMER. Polar coronal holes are recognized as the sources of the fast solar wind. Panels $b 1$ and $b 2$ : An active region imaged by EIS in the spectral line of Fe XII at $195.12 \AA$. There is mounting evidence that outflows from the edges of active regions contribute to the slow solar wind. Panels $a 1$ and $b 1$ show integrated line radiances. Panels $a 2$ and $b 2$ show Doppler maps. Blue indicates an outflow; red a downflow along the observer line-of-sight. Note that the extensive outflows occur in regions that are faint in Ne VIII in the polar coronal hole and in Fe XII in the active region.

$83^{\circ}$ angle) resolution better than $2^{\prime \prime}$ (taking advantage of the $\approx 7^{\circ}$ tilt of the solar rotational axis). The Solar-C location in the ecliptic plane makes on-disk studies of the polar regions more difficult but it is ideal for studies of the plasma conditions above the limb. This makes the mission highly complementary to Solar Orbiter, which will observe the poles from about $34^{\circ}$ above the ecliptic. Solar Orbiter will study the dynamics and evolution of on-disk polar regions (e.g., roots of plumes) while Solar-C will look at the above limb effects of such dynamics and evolution.

Hinode observations have revealed persistent flows (see Figure 7b) at the edges of active regions [see, 33]. These coronal outflows are in part possibly related to open field regions connected into the heliosphere [e.g., 34] and could provide up to $25 \%$ of the slow solar wind in some cases. They are therefore an important feature to be studied in conjunction with close-by in-situ observations from Solar Orbiter and Solar Probe+. At present it is impossible to connect these flows with transition region and chromospheric structures, due to incomplete temperature coverage by Hinode/EIS and its limited spatial resolution. This can be achieved with LEMUR. 
Table 2 Science traceability matrix connecting LEMUR science goals to Solar-C goals.

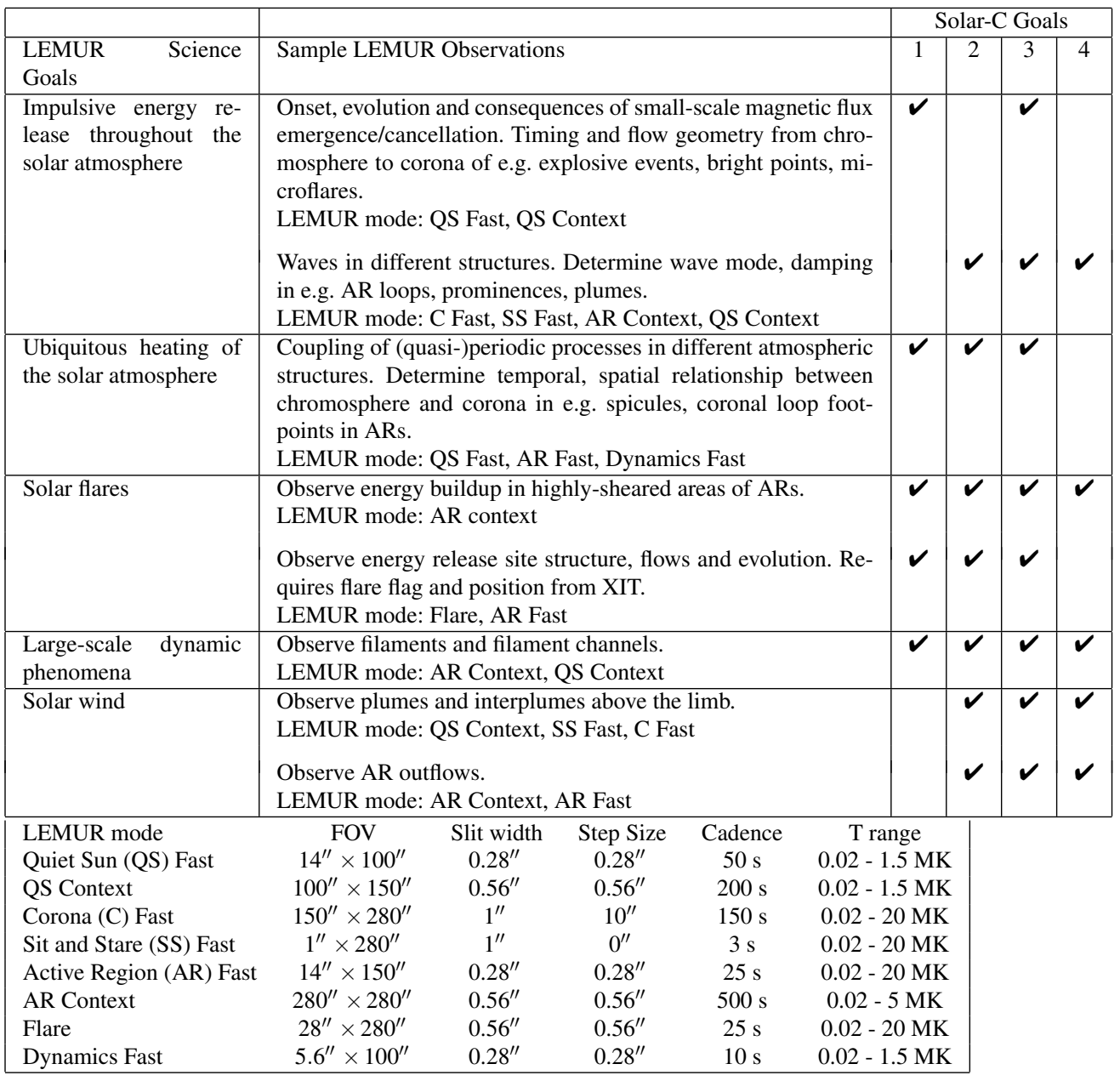

\subsection{LEMUR Performance Requirements}

It is clear from the science discussion above that to understand the phenomena to the depth that we desire, we need an imaging spectrometer with much more sensitivity, temperature coverage, and higher spatial and spectral resolution than is available from all current instrumentation. One way to think about the design requirements for LEMUR is to devise a set of basic observing sequences that achieve the science goals we have outlined. Table 2 contains a list of desired observations that address many of the LEMUR and Solar-C science goals along with the kind of observing program that would acquire the data necessary to achieve the science goal. These observations rely on a small set of observing sequences listed at the bottom of the table. Collectively, they define the design requirements for the instrument. 
Consideration of these desired observations, as well as many others, and of the measured sizes, speeds, lifetimes, and regions of propagation of the important solar phenomena using available data has led to the design parameters of LEMUR listed in Table 3. A large primary mirror is needed to achieve high sensitivity and spatial resolution. The high sensitivity enables high time resolution. The mirror coatings are designed to provide high reflectivity over the full wavelength range covered by LEMUR. Image motion compensation is desirable due to the high spatial resolution which requires high fidelity in tracking moving features. The optical parameters are also chosen to maximize spectral resolution in order to detect the small flows that have been seen and that provide valuable insights into the observed phenomena.

Table 3 LEMUR instrumental requirements

\begin{tabular}{ll}
\hline Field & Required value \\
\hline Spatial resolution & $\leq 0.28^{\prime \prime}$ \\
Spectral resolution & $\lambda / \Delta \lambda 17000$ to 32000 \\
Doppler shift accuracy & $\leq 2 \mathrm{~km} \mathrm{~s}^{-1}$ \\
Doppler width accuracy & $\leq 5 \mathrm{~km} \mathrm{~s}^{-1}$ \\
Temperature coverage & 0.01 to $20 \mathrm{MK}$ \\
Field-of-view & slit length $280^{\prime \prime}$ \\
raster coverage & $300^{\prime \prime}$ (w/o re-pointing) \\
Exposure times & $\leq 10 \mathrm{~s}\left(0.28^{\prime \prime}\right.$ sampling) \\
& $\leq 1 \mathrm{~s}\left(1^{\prime \prime}\right.$ sampling) \\
Mirror micro-roughness & about $3 \AA$ rms or better \\
\hline
\end{tabular}

The requirements summarized in Table 3 can be met technologically by designing an instrument with as few optical elements as possible. The EIS spectrometer, a two-optic system, provides a good baseline design for LEMUR. In order to achieve the resolutions and temporal requirements stated above, the telescope mirror needs to be substantially larger than the SUMER (rectangular, $9 \times 13 \mathrm{~cm}^{2}$ ) and EIS (circular, $15 \mathrm{~cm}$ diameter) mirrors. Further, a combination of a EUV multilayer (i.e., $\mathrm{Mo} / \mathrm{Si}$ ) and a coating such as $\mathrm{B}_{4} \mathrm{C}$ is necessary to cover the large wavelength range needed to probe the required temperature range. For the same reason the instrument will not have a front filter. Thus, at shorter wavelengths, LEMUR will produce spectra and images completely free of the diffraction ghosts emanating from the mesh supporting the entrance filter. Low-scattering optics are necessary in order to explore faint reconnection outflow regions, coronal holes, and to make off-limb observations at up to 1.5 solar radii. Accessing structures above the limb at the nominal spatial resolution of 0.28 " (e.g., loop systems) requires a pointing mechanism moving the main mirror over a spherical surface (like in SUMER). Precise co-alignment must be achieved with other Solar-C on-board instruments. The co-alignment must meet the high spatial resolution of the spectrometer and requires a slit camera (slit imaging assembly) imaging the chromosphere at about $0.3^{\prime \prime}$ resolution. 


\section{Mission profile: orbit and thermal considerations}

The preferred orbit is an inclined geosynchronous orbit (GSO, altitude $36000 \mathrm{~km}$, inclination $<30^{\circ}$, period 1 day, similar to SDO), with a Sun-synchronous polar orbit (altitude 680 to $800 \mathrm{~km}$, inclination 97 to $98^{\circ}$, period $98 \mathrm{~min}$, similar to Hinode) as backup. The GSO is preferred because of the more stable thermal conditions and of the long periods of contact that can provide high volume telemetry and allow near real time scientific operations, which are required to meet science requirements.

The very favorable thermal environment provided by the GSO helps to meet LEMUR's requirements by implementing a passive, cold biased thermal system, optimized for insusceptibility to external heat sources. The adverse effect of Earthshine and albedo on LEMUR can be minimized by optimal radiator placement and orientation $^{\text {iii }}$. In GSO, for several weeks daily eclipsing episodes of up to 70 min occur twice a year, when the $\mathrm{S} / \mathrm{C}$ crosses the ecliptic. Compensation heaters will minimize the post eclipse recovery time to less than $1 \mathrm{~h}$.

An analysis based on a simplified 16-node simplified thermal mathematical model has shown that the thermal concept is uncritical, leaving room for adaptations during instrument design maturation. It should be noted that optical performance reasons do not allow conductive cooling, so that the mirror will run hot. The mirror of LEMUR will be figured and polished for an elevated constant operational temperature of $\sim 85^{\circ} \mathrm{C}$, a concept that optimizes figure stability and minimizes contamination. The feasibility of this concept is demonstrated by the SUMER primary mirror, successfully operating at $\sim 83^{\circ} \mathrm{C}$.

\section{LEMUR module design, performance characteristics, and key resources}

The LEMUR design is driven by the instrument requirements given in Table 3 and meets them with ample margin.

\subsection{LEMUR module description}

The LEMUR module consists of a telescope unit and a spectrograph unit. A physical block diagram of the LEMUR module is given in Figure 8.

The module will be mounted with isostatic mounting legs to a mechanical interface provided by the Solar-C spacecraft. The mounting will be designed to provide thermal independence from the spacecraft. The Telescope unit structure is the main support structure of the LEMUR module and has provisions to mount the Spectrograph Structure. The opto-mechanical layout of the LEMUR system is shown in Figure 9. The telescope unit consists of a front door assembly, the single-mirror telescope assembly (an off-axis parabola with pointing capabilities), the guide telescope, the telescope electronics, the heat rejection assembly and the deflector plate assembly.

\footnotetext{
iii In low Earth orbit, this effect would be much more severe and compensation heaters would be needed to dampen short-term temperature excursions.
} 


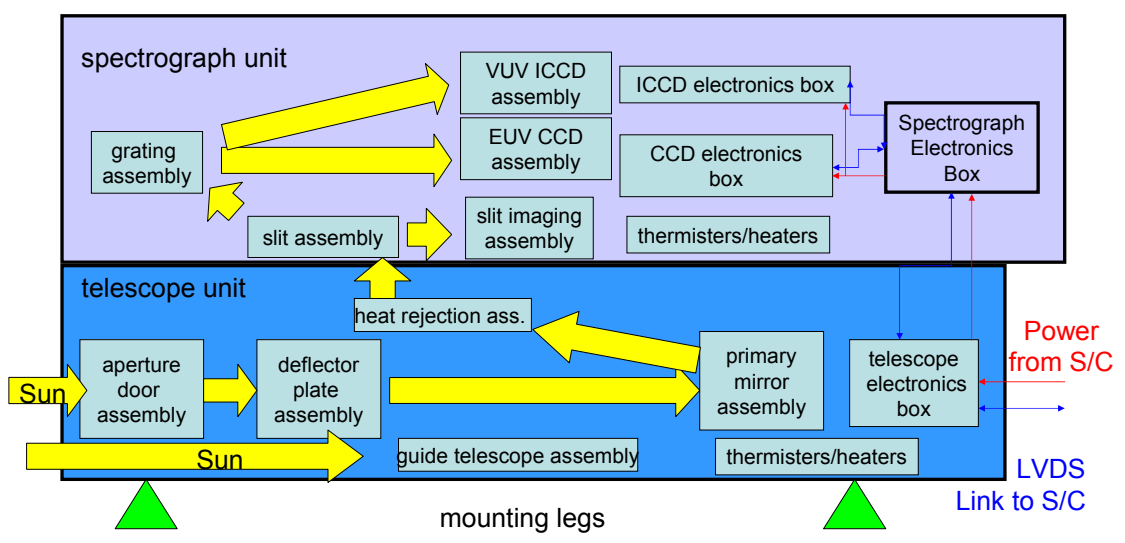

Fig. 8 LEMUR physical block diagram showing major LEMUR components and digital communication links. The telescope electronics box, provides power and digital interfaces (via low-voltage differential signaling, LVDS links) to the S/C on one side and to the spectrograph electronics box on the other.

The spectrograph unit houses the slit assembly, the slit imaging assembly, the grating assembly, the focal plane assemblies, CCD electronics box, Intensified CCD (ICCD) electronics box and detector radiator assembly. The Telescope unit and the Spectrograph unit are designed to be assembled and tested separately. They can be mated with each other for final testing and calibration before integration to the spacecraft (S/C).

LEMUR telescope unit - The telescope unit provides the solar image in its focal plane, the entrance to the spectrograph unit, and provides image stabilization and pointing capabilities. The mirror assembly consists of the telescope mirror and a combined pointing and raster scanning mechanism. It is used to compensate for residual image motion and to provide coarse pointing of the telescope, as well as stepwise scanning to produce raster images. The mirror produces an image in the center of the focal plane of the parabola, to feed the spectrograph unit. Before the slit plane, a preslit heat rejection mirror collects more than $95 \%$ of the heat of the solar image to be directed to a heat dump, whereas only a small fraction of the image will pass towards the slit. The open telescope design requires a front door to protect the instrument during ground and launch operations. Electrostatic deflection plates, close behind the front door in the telescope baffle, protect the mirror coating from incoming solar wind particles. A guide telescope provides a signal for image motion stabilization.

LEMUR spectrograph unit - The spectrograph unit accepts the light from the telescope passing through the entrance slit towards the grating and the detector assemblies. The slit assembly consists of slits of five widths mounted on a translation stage mechanism used to select the slit for a given observing program. The slit assembly also contains a focus adjustment capability. The grating assembly consists of two 


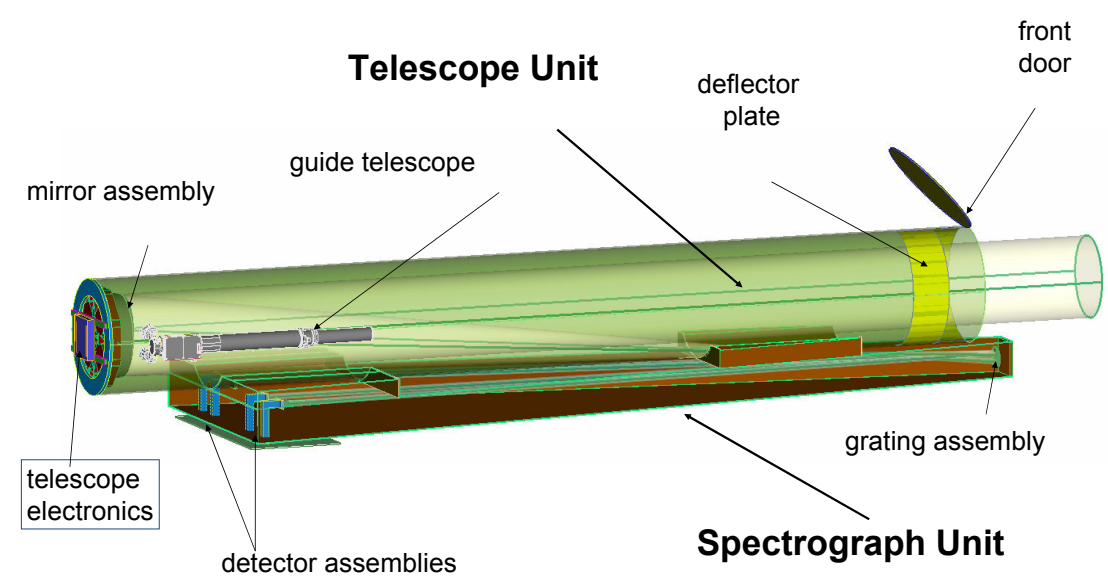

Fig. 9 The opto-mechanical layout of LEMUR. Incoming solar light enters the instrument from the right, trough the front door.

gratings, one for the 170 to $210 \AA$ EUV band (SW) and the other for three bands at wavelengths above $480 \AA$ (LW-1: 695 to $815 \AA$, LW-2: 965 to $1085 \AA$, LW-3: 1150 to $1270 \AA$ ). $\mathrm{LW}-2$, and LW-3 are expected to record some particularly intense second order lines. The grating assembly is mounted on a focusing mechanism. Each grating is ruled, figured and coated to optimize the optical performance of its respective channel. The dispersed slit images are projected onto the focal plane cameras, the SW camera with a thin foil filter and the three solar-blind intensified cameras, LW-1, LW-2, and LW-3.

Table 4 summarizes unit and assembly key characteristics. Description and design characteristics of the LEMUR optics, detectors, structure and electronics are provided in the sections below.

\subsection{LEMUR detailed design}

The LEMUR design follows that pioneered by the SUMER/SOHO and EIS/Hinode instruments. The telescope unit is contained in a precision manufactured, low expansion, light weight CFRP structure. Behind the entrance door is a long optical baffle completed by an aperture stop with optical power (reflective and curved) for stray light reduction and heat management. Inside the telescope baffle will be a deflector plate assembly, a plate arrangement to protect the primary mirror from ambient charged particles. A static voltage is applied across the two plates such that solar wind particles will be deflected from their path towards the mirror. The mirror will be mounted to a mirror assembly with radiative coupling to a radiator for cooling. The mirror assembly provides mechanisms for coarse pointing and fine rastering movements. The coarse pointing provides movement on a spherical surface around 
the focal center in north-south and east-west directions with a range of $3000^{\prime \prime}$. The fine pointing capability allows image motion stabilization and raster scanning in a $300^{\prime \prime}$ range. The telescope electronics box provides control of the telescope mechanisms and sensors and handles the signal of the guide telescope. It provides the digital and power interfaces to the $\mathrm{S} / \mathrm{C}$ on one side and to the spectrograph electronic box on the other. During operation of the LEMUR module, it is controlled by the spectrograph electronic box. The off-axis paraboloid telescope projects the solar image onto a slit assembly. Before the slit is a heat rejection assembly, a curved mirror to reject all but a $250^{\prime \prime} \times 400^{\prime \prime}$ patch of the solar image. The rejected light is transferred to a heat dump or reflected out to space. The heat rejection assembly belongs to the telescope unit while the slit assembly is part of the spectrograph unit. The spectrograph structure is a precision manufactured CFRP honeycomb panel structure, to be mounted on the telescope unit.

The slit assembly contains a double mechanism to select one of several slits on a slit substrate and a linear translation to move the slit into best focus of the telescope. Several slits with different widths will be available (e.g., $0.14^{\prime \prime}, 0.28^{\prime \prime}, 0.56^{\prime \prime}, 1^{\prime \prime}$ and $5^{\prime \prime}$, the latter to be used mostly for calibration purposes).

The slit jaws reflect the solar image passing through the pre-slit towards the slit imaging assembly: a small monochromatic camera with relay optics to provide a context image of the slit position in a $200^{\prime \prime} \times 300^{\prime \prime}$ field of view and a resolution of $0.3^{\prime \prime}$. The wavelength band will be selected to fulfill co-registration requirements with the other SOLAR-C instruments.

The spectrograph splits the beam into the SW and LW channels by the two gratings which are tilted by a small angle oppositely with respect to the optical dispersion plane. Thus, each channel incorporates a highly corrected, single-grating imaging spectrograph. First and second order spectral lines are chosen by a special selective photocathode coating on the LW detectors (see below). The gratings are housed on a linear translation mechanism with $2 \mathrm{~cm}$ range for focus adjustment, whenever necessary, to provide dispersed images of the entrance slit in the detector plane. The two focal plane assemblies are imaging arrays, highly efficient for each spectral passband. The SW detector is constructed with two butted, back-side thinned CCDs ( $2 \mathrm{k}$ $\times 2 \mathrm{k}$ format) and a thin metal foil filter to block visible radiation. The LW focal plane arrays are three CCD units coupled with a microchannel plate (MCP) intensifier. The spectrograph electronic box (E-box) drives all mechanisms and cameras of the spectrograph units and provides commands to the telescope E-box. All design characteristics of the LEMUR module are summarized in Table 4.

\subsubsection{LEMUR optical design}

The LEMUR optical design follows the two element design pioneered by the EIS instrument aboard Hinode. The telescope mirror is a $30 \mathrm{~cm}$ diameter section of a parabola, placed $22.5 \mathrm{~cm}$ off axis, with $360 \mathrm{~cm}$ focal length. The mirror produces a plate scale of $17.5 \mu \mathrm{m} /{ }^{\prime \prime}$ at the location of the spectrograph entrance slit. The imaging performance of the telescope is shown in Figure 10. The optical characteristics of the telescope are given in Table 4. 
Table 4 LEMUR module design characteristics

\begin{tabular}{|c|c|}
\hline Unit/assembly & Nominal value/description \\
\hline \multicolumn{2}{|r|}{ Telescope unit } \\
\hline Telescope type & Single-mirror off-axis paraboloid \\
\hline Optical characteristics & $\begin{array}{l}30 \mathrm{~cm} \text { diameter, } 360 \mathrm{~cm} \text { focal length, } \lambda / 75 \mathrm{rms} \text { figure, } \\
<3 \AA \text { rms micro-roughness, broadband VUV coating } \\
\text { low-expansion substrate (Zerodur or ULE) }\end{array}$ \\
\hline Deflector plate & Two plates with static HV supply \\
\hline Baffles/aperture stop & Built as heat rejection mirror \\
\hline Heat rejection pre-slit & Curved mirror in front of the slit, $250^{\prime \prime} \times 400^{\prime \prime}$ central aperture \\
\hline Telescope pointing & $\begin{array}{l}\text { Coarse pointing capability: } 3000^{\prime \prime} \text { range in pitch and yaw } \\
<1^{\prime \prime} \text { steps with } 10^{\prime \prime} \text { accuracy. } \\
\text { Fast raster/fine pointing mechanism: range } 300 " \\
\text { image motion in pitch and yaw, } 0.028^{\prime \prime} \text { digitization, } \\
>10 \mathrm{~Hz} \text { response }\end{array}$ \\
\hline Guide telescope & $<0.05^{\prime \prime}$ accuracy at $10 \mathrm{~Hz}$, range $>2000^{\prime \prime}$ \\
\hline \multirow{2}{*}{ Telescope E-box } & Control of telescope mechanisms and sensors \\
\hline & Digital and power interfaces to the S/C \\
\hline Front door & Reclosable front door \\
\hline Telescope structure & Precision manufactured CFRP structure \\
\hline \multicolumn{2}{|r|}{ Spectrograph unit } \\
\hline Spectrograph design & Ellipsoidal variable line space (EVLS) gratings \\
\hline Slit assembly & $\begin{array}{l}\text { Linear translation, } 5 \text { slits on a slit plate, } 0.14^{\prime \prime}, 0.28^{\prime \prime}, 0.56^{\prime \prime}, 1^{\prime \prime}, 5^{\prime \prime} \\
>2 \mathrm{~cm} \text { focus adjustment along telescope chief ray } \\
\text { Slit jaws reflective to feed the slit imaging assembly }\end{array}$ \\
\hline Grating assembly & $\begin{array}{l}\text { Houses the } S W \text { and LW gratings } \\
>2 \mathrm{~cm} \text { focus adjustment along telescope chief ray }\end{array}$ \\
\hline CCD focal plane assembly & $\begin{array}{l}\text { SW CCD with radiative cooling, } 2 \text { butted } 2048 \times 2048 \\
13.5 \mu \mathrm{m} \text { pixel with shutter mechanism, thin metal foil filter }\end{array}$ \\
\hline ICCD focal plane assembly & $\begin{array}{l}3072 \times 2048,20 \mu \mathrm{m} \text { pixel } \mathrm{LW} \text { ICCD } \\
\text { bare and CsI or } \mathrm{KBr} \text { coated MCP } \\
\text { protective vacuum door (optional) }\end{array}$ \\
\hline CCD E-box & Controls and reads the CCD detectors \\
\hline ICCD E-box & $\begin{array}{l}\text { Controls and reads the ICCD detectors, } \\
\text { provides MCP HV }\end{array}$ \\
\hline Slit imaging assembly & $\begin{array}{l}\text { Relay optics with }>200^{\prime \prime} \times 300^{\prime \prime} \text { FOV } \\
<0.3^{\prime \prime} / \text { pixel plate scale, CCD detector }\end{array}$ \\
\hline Spectrograph structure & Precision manufactured CFRP honeycomb panel structure \\
\hline Spectrograph E-box & TM and TC handling, data processing, camera operations \\
\hline
\end{tabular}

The LEMUR mirror coating is designed to have high normal-incidence reflectance in the VUV wavelength ranges 170-210 $\AA$ and $>480 \AA$. The LW channel ( $>480 \AA$ ) waveband is reflected from a top $\mathrm{B}_{4} \mathrm{C}$ layer. The $\mathrm{SW}$ channel wavelengths penetrate the $\mathrm{B}_{4} \mathrm{C}$ layer and the $170-210 \AA$ wavelengths are reflected by a multilayer interference coating consisting of alternating layers of $\mathrm{Si}$ and $\mathrm{Mo}$, traditional materials with extensive flight heritage. A unique reflective coating combination will be chosen for the entire area of the mirror as different combinations of coatings over different areas of the mirror might stress it and degrade its optical quality. Shown in Figure 11 is the reflectance calculated for three assumed $\mathrm{B}_{4} \mathrm{C}$ top layer thicknesses $(50 \AA, 100 \AA$, and $200 \AA$ ). The trade-off is that increasing the $\mathrm{B}_{4} \mathrm{C}$ thickness has the beneficial effect of 


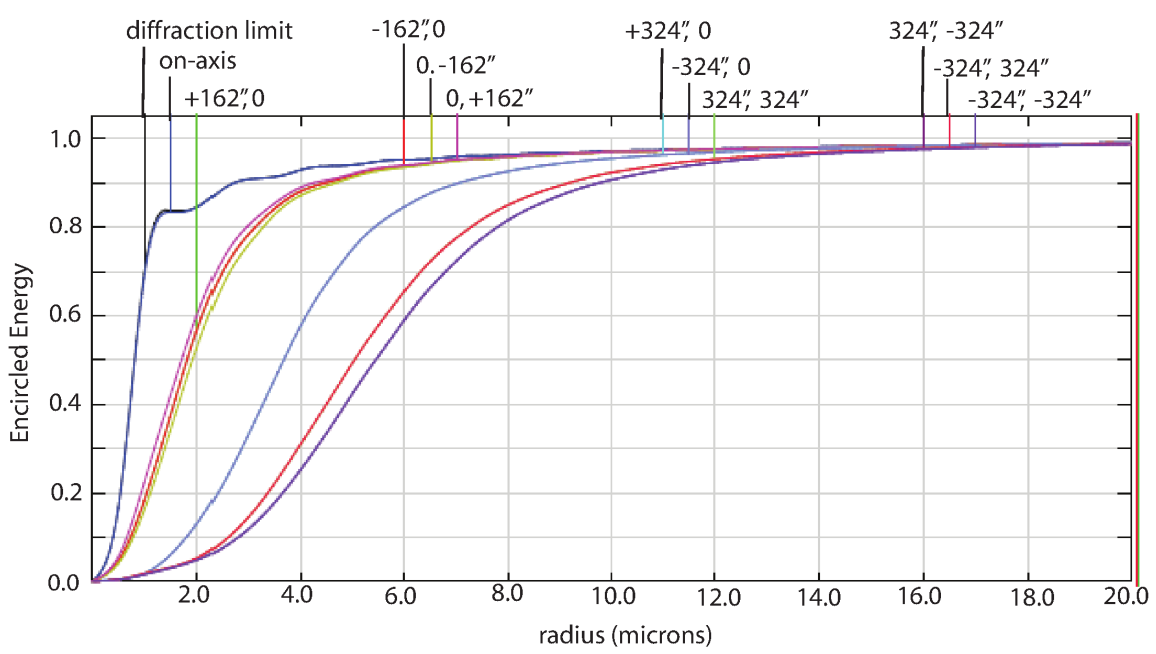

Fig. 10 Imaging performance of the LEMUR telescope. Encircled energy radius in microns are given for the off-axis paraboloid mirror at $1216 \AA$ for on-axis, \pm 160 , and \pm 320 arcsec field angles.
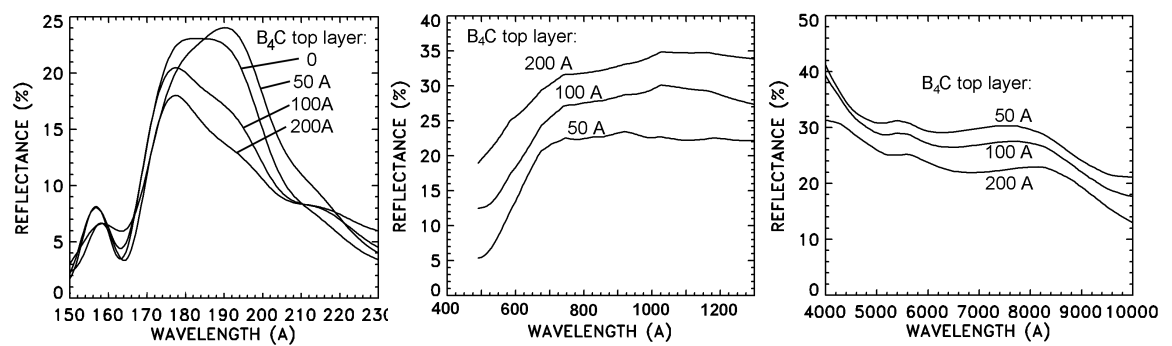

Fig. 11 LEMUR broadband Mo/Si multilayer mirror coating with $\mathrm{B}_{4} \mathrm{C}$ top layer with thicknesses of $50 \AA$, $100 \AA$, and $200 \AA$. The reflectance is shown for the SW band (left), LW band (middle), and the Vis-NIR range (right).

increasing the $>480 \AA$ reflectance but reduces the reflectance at shorter wavelength. The visible and near-IR reflectance is also shown since it affects the temperature of the mirror that accepts the solar heat load and also affects possible scattered out-ofband light. The mirror is required to have micro-roughness of $3 \AA$ rms or better ${ }^{\mathrm{iv}}$, significantly improving (of a factor $\approx 4$ ) stray light performances with respect to SUMER ( $6 \AA$ rms, mid-frequency error).

The slit selects a portion of the solar image and passes it onto the SW and LW gratings. Similar to $\mathrm{CDS} / \mathrm{SOHO}$, these two grating halves are mounted in close prox-

\footnotetext{
iv Mirrors with $<2 \AA$ rms micro-roughness are routinely produced by companies such as Zeiss for EUV lithography. Moreover, at longer wavelengths, samples with $<2 \AA$ rms micro-roughness have been produced for the SPICE instrument to be flown on Solar Orbiter.
} 
Table 5 LEMUR spectrograph optical prescription

\begin{tabular}{lll}
\hline \multicolumn{2}{c}{ SW waveband } & \multicolumn{1}{c}{ LW wavebands } \\
\hline Spectral ranges & $170-210 \AA$ & $\begin{array}{l}1^{\text {st }} \text { order: } 695-815 \AA, 965-1085 \AA, 1150-1270 \AA \\
2^{\text {nd }} \text { order: } 482-542 \AA, 575-635 \AA\end{array}$ \\
Dispersion & 4200 grooves $/ \mathrm{mm}$ & 1200 grooves $/ \mathrm{mm}$ \\
& $280 \mathrm{~cm}$ grating to detector & $414.8 \mathrm{~cm}$ grating to detector \\
& $10 \mathrm{~m} \AA /$ pixel & $40 \mathrm{~m} \AA /$ pixel \\
Plate scale & $0.14^{\prime \prime} / 13.5 \mu$ m pixel & $0.14^{\prime \prime} / 20 \mu \mathrm{m}$ pixel \\
Slit length & $280^{\prime \prime}(2000$ pixels $)$ & $280^{\prime \prime}(2000$ pixels $)$ \\
Magnification & 5.5 & 8.1
\end{tabular}

imity. The gratings are figured and coated to optimize the image quality and efficiency of each passband. The highly corrected off-axis elliptical gratings with variable line spacing give excellent image quality at the focal plane across all wavelength ranges. Table 5 gives the prescriptions of the optics and the spectrograph characteristics.

The coating for each grating half is optimized for its particular waveband. The LW grating half is coated with $200 \AA$ of $\mathrm{B}_{4} \mathrm{C}$. The SW grating half is coated with $\mathrm{Mo} / \mathrm{Si}$ bilayers without the $\mathrm{B}_{4} \mathrm{C}$ top layer.

The combined telescope and SW spectrograph performance at the central wavelength of $185 \AA$ at the slit center and extreme ends of the slit is shown in Figure 12. The combined telescope and LW spectrograph performance is shown in Figure 13 for all wavelength ranges. The LEMUR performance improvements are the result of a remarkable extension in spectrograph design which incorporates an off-axis Ellipsoidal Variable Line Space (EVLS) grating to produce the necessary resolution on the critical spectral, spatial, and temporal scales. The key to the success of this ellipsoidal grating concept is to orient the ellipse so that its two foci lie at the slit and at the detector. The segment of the ellipse actually used by the grating is off its normal axis. The remaining parameters of the EVLS grating are optimized using ZEMAX. The high grating magnification and the two elements optical design allow LEMUR to reduce its size relative to a conventional spectrometer with similar spatial resolution by a factor between 3 and 5. EVLS gratings do not require new technology development.

\subsubsection{Focal plane assemblies}

The baseline detector is a CCD from the E2V 42 family which has extensive flight heritage. During the conceptual design phase, the LEMUR team will study various detectors including the radiation hardened APS detectors and electronics presently under development for the Solar Orbiter mission.

CCD focal plane assembly - Similar to Hinode/EIS, it consists of two backside thinned CCD detectors. These detectors are closely butted together for a minimal gap. The focal plane incorporates a $1000 \AA$ thick aluminum foil filter to reject the visible radiation. This camera assembly is equipped with a focal plane shutter. The CCDs 


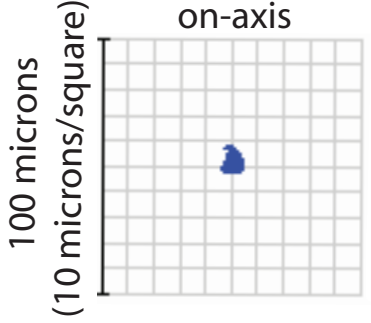

RMS radius 2.4 slit bottom

$0,130 "$

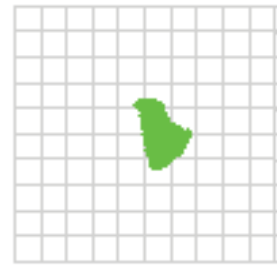

RMS radius 10.0 slit top

$0,-130^{\prime \prime}$

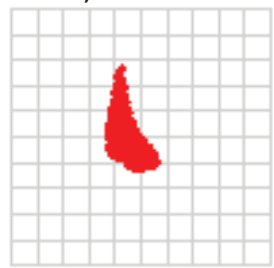

RMS radius 11.9

(RMS radius given in microns for SW channel, wavelength - 18.5nm)

Fig. 12 Combined telescope and SW spectrograph imaging performance at $185 \AA$ wavelength. Spots shown are for slit center, slit top and slit bottom position.

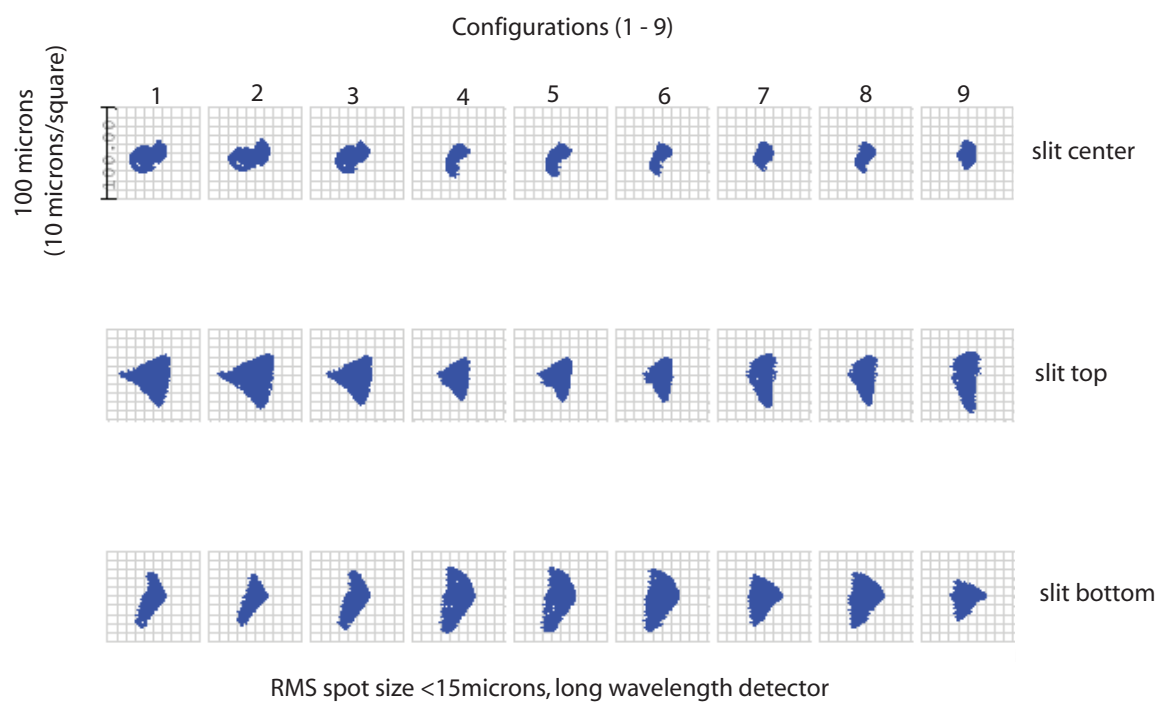

Fig. 13 Combined telescope and LW spectrograph imaging performance. Configurations 1 to 3 are for LW-1; configurations 4 to 6 are for LW-2; and configurations 7 to 9 are for LW-3. The top row of spots are at the center of the slit. The second and third rows are the spots at the end of the slit. The scale of the square field is $100 \mu \mathrm{m}$ on the side. Focal plane pixel size is $20 \mu \mathrm{m}$.

are thermally isolated and strapped to an external radiator to be cooled to $-60^{\circ} \mathrm{C}$.

ICCD focal plane assembly - The LW detectors consist of three independently operated intensified CCDs. ICCD operation is similar to previous ICCDs flown on SOHO/CDS and the SERTS instrument. The MCP intensifier provides high efficiency and solar blindness such that no focal plane filters are needed. Coupling between 


\begin{tabular}{|c|c|c|}
\hline Mechanism & Requirements/characteristics & Heritage \\
\hline Front door & $\begin{array}{l}\text { Re-closable door mechanism with fail-safe } \\
\text { redundant opening }\end{array}$ & $\begin{array}{l}\text { Secchi/HI on STEREO, } \\
\text { SUMER and LASCO on } \\
\text { SOHO }\end{array}$ \\
\hline Telescope pointing & $\begin{array}{l}\text { Coarse: linear translation via dual motor } \\
\text { stage along best image quality curve. } \\
\text { Fine: two axis rotation for rastering and for } \\
\text { image motion compensation using gimbal } \\
\text { structure, flexural mounts, flex pivots, and } \\
\text { voice coil drivers }\end{array}$ & $\begin{array}{l}\text { Design similar to previous } \\
\text { tip/tilt mirrors built by Ball } \\
\text { Aerospace or similar to the } \\
\text { SUMER pointing mechanism }\end{array}$ \\
\hline Slit select/focus & $\begin{array}{l}5 \text { slits on a linear translation mechanism } \\
>2 \mathrm{~cm} \text { focus adjustment along the chief } \\
\text { ray }\end{array}$ & SUMER/SOHO, EIS/Hinode \\
\hline Grating focus & $>2 \mathrm{~cm}$ linear grating translation & SUMER/SOHO, EIS/Hinode \\
\hline CCD shutter & $\begin{array}{l}\text { Direct driven shutter mechanism with opti- } \\
\text { cal encoding. }>4 \times 10^{7} \text { cycles }\end{array}$ & EIS/Hinode, others \\
\hline $\begin{array}{l}\text { ICCD detector door } \\
\text { (optional) }\end{array}$ & $\begin{array}{l}\text { One-shot door. Fail-safe actuator. Mechan- } \\
\text { ical GSE for door re-closure during ground } \\
\text { operations }\end{array}$ & HST and other missions \\
\hline
\end{tabular}

intensifier and CCD is made by fiber optic taper from a $20 \mu \mathrm{m} \mathrm{MCP}$ pixel to a 13.5 $\mu \mathrm{m}$ CCD pixel (1.48:1). The MCP will be coated with photocathode material of cesium iodide $(\mathrm{CsI})$ or potassium bromide $(\mathrm{KBr})$ in selective locations of the spectral ranges to enhance sensitivity of first-order lines against second-order lines that are, this way, largely suppressed. Five spectral ranges over LW-3 will be left uncoated to allow four selected $2^{\text {nd }}$ order lines of particular relevance to dominate over the first order lines. A fifth area of LW-3 will be left uncoated to reduce the extremely strong signal from the hydrogen Lyman $\alpha$ line. The MCP intensifier may be protected until scientific operations start by a protective door that can be opened in orbit and closed on demand. The CCDs will provide an array of $3072 \times 2048$ pixels with $20 \mu \mathrm{m}$ size. The exposures are electronically controlled by switching the MCP voltage and the CCD can be operated without mechanical shutter. The CCDs can be passively cooled to $-60{ }^{\circ} \mathrm{C}$. This requirement will be revisited during the initial design phase.

\subsubsection{Mechanisms}

The mechanism assemblies required by LEMUR for operation of all subsystems and their expected characteristics and heritage are summarized in Table 6.

\subsection{Radiometric Performance assessment}

The two element optical design of LEMUR (minimum number of reflections), the large aperture, the good reflectance of the optics and the high quantum efficiency of the detectors, all contribute to an unprecedented throughput. Figure 14 compares the count $\mathrm{s}^{-1}$ obtained from a solar active region by LEMUR with those from the 


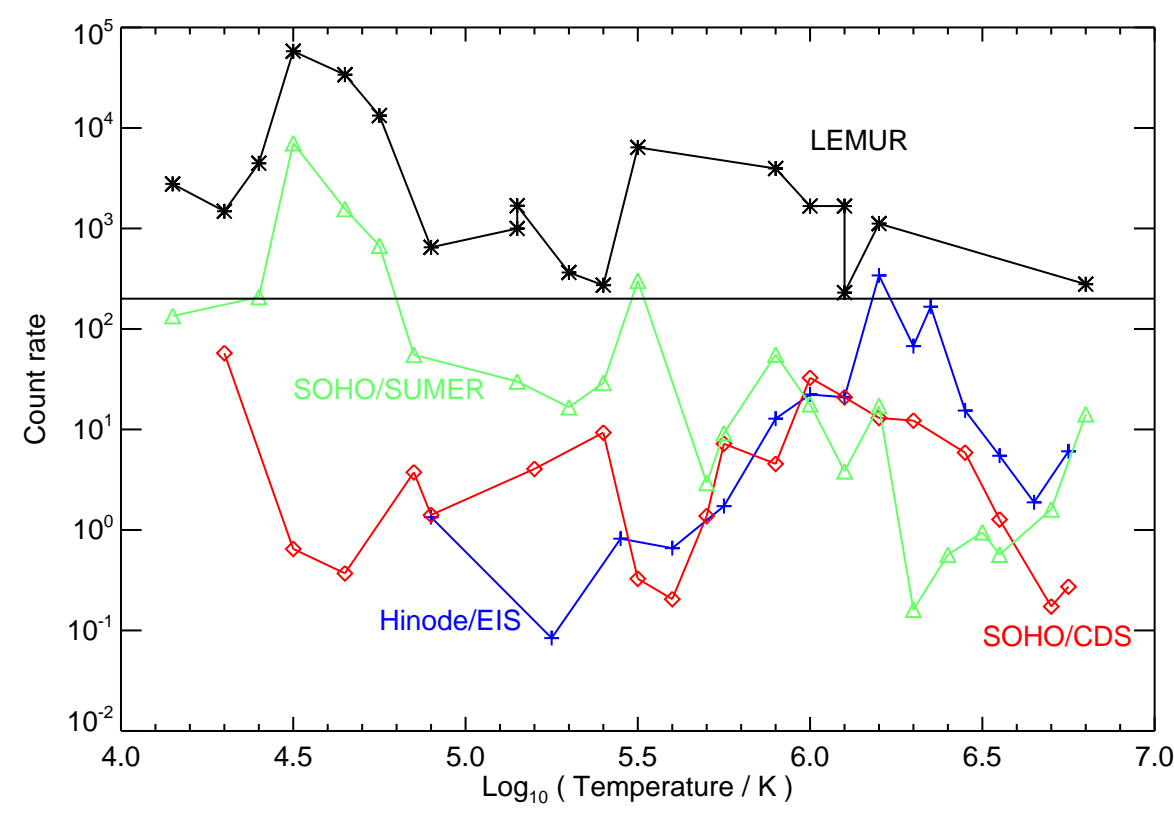

Fig. 14 Comparison of the LEMUR expected count-rates with those from CDS and SUMER on SOHO and EIS on Hinode. The $200 \mathrm{count} / \mathrm{s}$ level is indicated by the solid horizontal line.

currently available spectrometers on Hinode and SOHO. Considering that about 200 counts in a spectral line are needed to measure flows with a $<2 \mathrm{~km} \mathrm{~s}^{-1}$ accuracy, it is evident that only LEMUR can and will obtain spectra of sufficient quality at all temperatures to perform the studies defined in Table 2 and essential to address the science goals illustrated in Section 2.

\subsection{Summary of LEMUR nominal instrument resources}

A preliminary mass breakdown gives an estimated mass of $155 \mathrm{~kg}$ (15\% margin included) for LEMUR ( $85 \mathrm{~kg}$ for the telescope unit and $70 \mathrm{~kg}$ for the spectrograph unit). An estimate of the average dissipated power is of $68 \mathrm{~W}$ (including $24 \mathrm{~W}$ for operational heaters). Many of the resource allowances are derived from actual assemblies built for SERTS, EIS and SUMER. The expected volume required for LEMUR is $430 \times 40 \times 70 \mathrm{~cm}^{3}$. The predicted average data volume of LEMUR is of about 1.5 Mbps (after compression).

\subsection{Pointing and alignment requirements}

LEMUR will be precisely pointed to the Sun together with the Solar-C spacecraft. During a single exposure, the pointing must be maintained to within $\sim 0.05^{\prime \prime}$ rms to 
Table 7 Preliminary analysis results for LEMUR payload reference axis stabilization on the Solar-C S/C.

\begin{tabular}{ll}
\hline Time period & expected stability \\
\hline $0.5 \mathrm{~s}$ & $<0.3^{\prime \prime} 3 \sigma$ \\
$5 \mathrm{~s}$ & $<0.3^{\prime \prime} 3 \sigma$ \\
$1 \mathrm{~h}$ & $<2,0^{\prime \prime} 0$ to peak \\
Mission life & $<32 .^{\prime \prime} 0$ to peak \\
\hline
\end{tabular}

maintain an overall resolution of $0.28^{\prime \prime}$. The S/C Team at JAXA has conducted simulations of the spacecraft pointing performance with the Hinode pointing performance used as bench mark reference in these studies. At the present time, the LEMUR line of sight is expected to be within the values given in Table 7 .

The current S/C analysis indicates that LEMUR requires an active internal stabilization system. A comparison of the predicted performance of the LEMUR reference axis stability and the line of sight knowledge and stability requirements shows that modest line of sight correction is required within the LEMUR module.

This modest correction can be achieved using the combination of a fine sun sensor (guide telescope), image motion stabilization (articulated primary mirror) and internal references (slit imaging assembly). The primary control loop for line of sight stabilization is implemented using the output of the guide telescope to steer small corrective motions of the telescope mirror. Initial calculations show that this control loop can operate at $>10 \mathrm{~Hz}$ frequency and provides the required $0.15^{\prime \prime}(3 \sigma)$ stabilization. Spacecraft jitter above the control frequencies are expected to be similar to SOT/Hinode. The SUVIT science instrument on the SOLAR-C payload requires a more stringent stability of $0.015^{\prime \prime}$. The SUVIT instrument also incorporates an internal motion stabilization system.

\subsection{Special issues: radiometric calibration}

The LEMUR radiometric calibration concept uses the same approach successfully applied to calibrate the SOHO and Hinode VUV spectrographic instrumentation. Component efficiencies will be characterized over their entire area using existing facilities at synchrotron radiation laboratories. Both detector and optical efficiencies will be measured. The Telescope Unit itself will not need a full radiometric calibration. The end-to-end calibration of LEMUR (Telescope and Spectrograph Unit assembly) will be made using a VUV transfer source standard traceable to a primary source. Past experience demonstrates that an absolute radiometric calibration with uncertainties $<15 \%$ over the entire spectral range can be achieved. In-orbit flat-field response changes are monitored by placing the wide $5^{\prime \prime}$ slit in the focal plane and then moving the solar image at the slit in small increments while taking repeated exposures. Similar techniques were used successfully to obtain the flat-field response of CDSEIT-SUMER/SOHO, EIS/Hinode and TRACE. Lastly, LEMUR will collaborate with sounding rocket flights to update the instrument calibration throughout its mission 
whenever possible. A rigorous contamination control program will be implemented to maintain the instrument efficiency.

\subsection{Current heritage and Technology Readiness Level (TRL)}

LEMUR fully exploits extensive scientific and technical heritage in building large scale solar and spectroscopic instrumentation in Europe, the US and Japan. In fact, LEMUR is a larger scale version of instruments flown on previous missions (EIS aboard Hinode and SUMER aboard SOHO) and, as an entire instrument, is overall at TRL 7. The assemblies and electronics boxes comprising LEMUR have extensive space flight heritage.

\section{Summary and conclusions}

Our understanding of the outer solar atmosphere has been greatly improved by the successful solar missions launched in the past two decades. SOHO, Hinode, and SDO clearly show that the solar atmosphere is a very complex and dynamic environment where energy is transported and dissipated throughout all temperature regimes, with the magnetic field being the most important player. To make a decisive step forward in our understanding of the solar (and hence stellar) magnetized atmosphere it is now necessary a comprehensive and simultaneous investigation of all the temperature regimes, from $0.01 \mathrm{MK}$ up to the $20 \mathrm{MK}$ observed during flares. These measurements must be as simultaneous as possible, have matching spatial resolutions, and need to be coupled to measurements of the magnetic field in the photosphere and chromosphere. These are the drawing lines of the Japanese Solar C mission and its payload consisting of the three state-of-art instruments (here named SUVIT, XIT, and LEMUR), described in Section 2.

LEMUR (Large European Module for solar Ultraviolet Research), the subject of the present paper, provides the crucial link between the photospheric and chromospheric magnetic field and plasma characteristics obtained by the visible telescope and the high temporal and spatial resolution images of the corona provided by the $\mathrm{X}$-ray telescope. Its principal science requirement is to obtain spectroscopic observations with sufficient resolution to measure the flow and dissipation of energy from the top layers of the chromosphere into the transition region and corona, and to observe multi-million-degree flare plasmas. LEMUR will diagnose gas at all relevant temperatures with unprecedented spatial, spectral, and temporal resolution. LEMUR's payload specifications are summarized in Table 1.

LEMUR consists of two major components: a VUV solar telescope with a $30-\mathrm{cm}$ diameter main mirror and a focal length of $3.6 \mathrm{~m}$, and a focal-plane package composed of VUV single-grating spectrometers covering six carefully chosen wavelength ranges between $170 \AA$ and $1270 \AA$. The LEMUR slit covers $280^{\prime \prime}$ on the Sun with $0.14^{\prime \prime}$ per pixel sampling. In addition, LEMUR is capable of achieving a temporal resolution of $0.5 \mathrm{~s}$.

No major technology development is required to implement LEMUR. It is at technology readiness level (TRL) 7 or higher for all components. 
LEMUR has been proposed as a possible ESA-led contribution to the Solar C mission in the 2010 Call for M Class missions. It will enable the European scientific community to participate as a key partner in a mission that will revolutionize our understanding of the magnetized atmosphere of our own star.

\section{References}

1. J. Trujillo Bueno, in Magnetic Coupling between the Interior and Atmosphere of the Sun, ed. by S. S. Hasan \& R. J. Rutten (2010), p. 118

2. L.K. Harra, T. Sakao, C.H. Mandrini, H. Hara, S. Imada, P.R. Young, L. van Driel-Gesztelyi, D. Baker, ApJ676, L147 (2008)

3. P. Bryans, P.R. Young, G.A. Doschek, ApJ715, 1012 (2010)

4. C.J. Schrijver, M.L. De Rosa, Sol. Phys.212, 165 (2003)

5. G. Del Zanna, H.E. Mason, J. Cirtain, in SOHO-17, ESA Special Publication, vol. 617 (2006), ESA Special Publication, vol. 617

6. D.E. Innes, B. Inhester, W.I. Axford, K. Wilhelm, Nature386, 811 (1997)

7. L. Teriaca, D. Banerjee, J.G. Doyle, A\&A349, 636 (1999)

8. M.J. Aschwanden, L. Fletcher, C.J. Schrijver, D. Alexander, ApJ520, 880 (1999)

9. T. Wang, S.K. Solanki, W. Curdt, D.E. Innes, I.E. Dammasch, ApJ574, L101 (2002)

10. J.T. Mariska, K. Muglach, ApJ713, 573 (2010)

11. T. Wiegelmann, T. Neukirch, A\&A457, 1053 (2006)

12. B. De Pontieu, S.W. McIntosh, M. Carlsson, V.H. Hansteen, T.D. Tarbell, C.J. Schrijver, A.M. Title, R.A. Shine, S. Tsuneta, Y. Katsukawa, K. Ichimoto, Y. Suematsu, T. Shimizu, S. Nagata, Science 318, 1574 (2007)

13. J.W. Cirtain, L. Golub, L. Lundquist, A. van Ballegooijen, A. Savcheva, M. Shimojo, E. DeLuca, S. Tsuneta, T. Sakao, K. Reeves, M. Weber, R. Kano, N. Narukage, K. Shibasaki, Science 318, 1580 (2007)

14. B. De Pontieu, S.W. McIntosh, V.H. Hansteen, C.J. Schrijver, ApJ701, L1 (2009)

15. B. De Pontieu, S.W. McIntosh, M. Carlsson, V.H. Hansteen, T.D. Tarbell, P. Boerner, J. Martinez-Sykora, C.J. Schrijver, A.M. Title, Science 331, 55 (2011)

16. G. Del Zanna, H.E. Mason, A\&A406, 1089 (2003)

17. H.P. Warren, D.M. Kim, A.M. DeGiorgi, I. Ugarte-Urra, ApJ713, 1095 (2010)

18. A. Bhattacharjee, Y. Huang, H. Yang, B. Rogers, Physics of Plasmas 16(11), $112102(2009)$

19. J.K. Edmondson, S.K. Antiochos, C.R. DeVore, T.H. Zurbuchen, ApJ718, 72 (2010)

20. W. Manchester, IV, ApJ666, 532 (2007)

21. L.M. Green, B. Kliem, A.J. Wallace, ArXiv e-prints (2010)

22. A.A. van Ballegooijen, P.C.H. Martens, ApJ343, 971 (1989)

23. G.D.R. Attrill, L.K. Harra, L. van Driel-Gesztelyi, M.J. Wills-Davey, Sol. Phys.264, 119 (2010)

24. A.S. Krieger, A.F. Timothy, E.C. Roelof, Sol. Phys.29, 505 (1973)

25. K. Wilhelm, I.E. Dammasch, E. Marsch, D.M. Hassler, A\&A353, 749 (2000) 
26. C. Tu, C. Zhou, E. Marsch, L. Xia, L. Zhao, J. Wang, K. Wilhelm, Science 308, $519(2005)$

27. Y. Wang, Y. Ko, R. Grappin, ApJ691, 760 (2009)

28. G. Del Zanna, B.J.I. Bromage, H.E. Mason, A\&A398, 743 (2003)

29. L. Teriaca, G. Poletto, M. Romoli, D.A. Biesecker, ApJ588, 566 (2003)

30. A.H. Gabriel, F. Bely-Dubau, P. Lemaire, ApJ589, 623 (2003)

31. D. Banerjee, G.R. Gupta, L. Teriaca, ArXiv e-print 1009.2980 (2010)

32. S. Tsuneta, K. Ichimoto, Y. Katsukawa, B.W. Lites, K. Matsuzaki, S. Nagata, D. Orozco Suárez, T. Shimizu, M. Shimojo, R.A. Shine, Y. Suematsu, T.K. Suzuki, T.D. Tarbell, A.M. Title, ApJ688, 1374 (2008)

33. G.A. Doschek, H.P. Warren, J.T. Mariska, K. Muglach, J.L. Culhane, H. Hara, T. Watanabe, ApJ686, 1362 (2008)

34. G. Del Zanna, G. Aulanier, K. Klein, T. Török, A\&A526, A137+ (2011) 